\title{
Local Optimization based Segmentation of Spatially-Recurring, Multi-Region Objects with Part Configuration Constraints
}

\author{
Masoud S. Nosrati, Student Member, IEEE, Ghassan Hamarneh, Senior Member, IEEE ,
}

\begin{abstract}
The level set framework has been a popular medical image segmentation technique for many years due to its several advantages, such as parametrization independence, ease of implementation, extendibility from a curve in 2D to higher dimensions, and automatic handling of topological changes. However, existence of noise, low contrast and objects complexity in medical images cause many segmentation algorithms (including level set-based methods) to fail. Incorporating prior knowledge into image segmentation algorithms has proven useful for obtaining more accurate and plausible results. Two important constraints, containment and exclusion of regions, have gained attention in recent years mainly due to their descriptive power and intuitive definitions. In this paper, we augment the level set framework with the ability to handle these two intuitive geometric relationships, containment and exclusion, along with a distance constraint between boundaries of multi-region objects. Level set's important property of automatically handling topological changes of evolving contours/surfaces enables us to segment spatiallyrecurring objects (e.g. multiple instances of multi-region cells in a large microscopy image) while satisfying the two aforementioned constraints. In addition, the level set approach gives us a very simple and natural way to compute the distance between contours/surfaces and impose constraints on it. The downside, however, is a local optimization framework in which the final segmentation solution depends on the initialization. In fact, here, we sacrifice the optimizibility (local instead of global solution) in exchange for lower space complexity (less memory usage) and faster runtime (especially for large microscopic images) as well as no grid artifacts. Nevertheless, the result from validating our method on synthetic and several biomedical applications, mainly on multi-region cell segmentation in microscopy images and cardiac segmentation in MR images, showed the utility and advantages of this augmented level set framework (even with fully automatic or rough initialization that is distant from the desired boundaries). We also compared our framework with its counterpart methods in the discrete domain and reported the pros and cons of each of these methods in terms of metrication error and efficiency in memory usage and runtime.
\end{abstract}

Index Terms-Segmentation, level set, local optimization, geometrical constraints, spatially-recurring, containment, exclusion, distance constraint, microscopy, cardiac segmentation, left/right ventricle.

\section{INTRODUCTION}

B IOMEDICAL image segmentation is at the crux of a variety of biomedical imaging applications, such as computer aided diagnosis, therapy planning and delivery, computer aided interventions, and in the image analysis and

Masoud S. Nosrati and Ghassan Hamarneh are with Medical Image Analysis Lab, School of Computing Science, Simon Fraser University, Canada e-mail: $\{$ smn6,hamarneh $\} @$ sfu.ca quantification of histological data. Despite great advances in image segmentation, the accurate automatic (or even semiautomatic) partitioning of biomedical images with complex scenes and objects remains a challenging problem. Many attempts have been made to incorporate prior knowledge into the task of segmentation, since inclusion of shape, appearance and topological priors have proven useful for obtaining more accurate and plausible segmentation results.

Many objects in biomedical images consist of multiple regions, where each region has a meaningful geometric relationship, or interaction, with other regions of the object. For example, in histology and microscopy images, each cell consists of a cell membrane, nucleus and nucleolus, where the cell membrane contains the nucleus, and the nucleus contains nucleolus. These interactions between an object's regions have often been ignored in microscopic histology image segmentation or enforced via some ad-hoc post-processing (e.g. via parameter sensitive morphological operations or thresholding) [1], [34], [66], [68].

The majority of state-of-the-art image segmentation methods are formulated as optimization problems, i.e. energy minimization or maximum-a-posteriori estimation, mainly because of their: 1) formal and rigorous mathematical formulation, 2) availability of the mathematical tools for optimization, 3) capability to incorporate multiple (competing) criteria as terms in the objective function, 4) ability to quantitatively measure the extent by which a method satisfies the different criteria/terms, and 5) ability to examine the relative performance of different solutions.

In energy-based segmentation problems there is a trade off between: fidelity and optimizability [40], [61]. Fidelity describes how faithful the energy function is to the data and how accurate it can model intricate problem details. Optimizability determines how easily we can optimize the objective function and attain the global optimum. Generally, the better the objective function models the problem, the more complicated the objective function becomes and the harder it is to optimize. If we instead sacrifice fidelity to obtain a globally optimizable objective function, the solution might not be accurate enough for our segmentation purpose.

In this work, we move toward improving the fidelity in the multi-region level set formulation and opt for ensuring the objective function is flexible enough (even if it is non-convex) to accurately capture the intricacies of the multi-region objects segmentation problem.

In the image segmentation literature, improving objective 
Table I: A comparison of certain features of commonly used state-of-the-art methods and our proposed method.

\begin{tabular}{|c|c|c|c|c|c|c|c|c|}
\hline Method & ن & 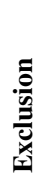 & 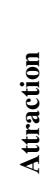 & 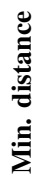 & 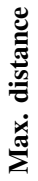 & 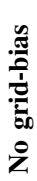 & 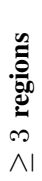 & 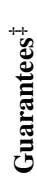 \\
\hline Wu 2011 [67] & $\checkmark$ & $x^{\circ}$ & $\checkmark$ & $\checkmark$ & $\checkmark$ & $x$ & $x$ & $\checkmark$ \\
\hline Zhao 1996 [71] & $x$ & $\checkmark$ & $x$ & $x$ & $x$ & $\checkmark$ & $\bar{\checkmark}$ & $\bar{\checkmark}$ \\
\hline Samson 2000 [55] & $x$ & $\checkmark$ & $x$ & $x$ & $x$ & $\checkmark$ & $\checkmark$ & $x$ \\
\hline Li $2006[28]$ & $\checkmark$ & $x$ & $\checkmark$ & $\checkmark$ & $\checkmark$ & $x$ & $\checkmark$ & $\checkmark$ \\
\hline Zeng 1998 [70] & $\checkmark$ & $x$ & $\checkmark$ & $\checkmark$ & $\checkmark$ & $\checkmark$ & $x$ & $x$ \\
\hline Goldenberg 2002 [21] & $\sqrt{ }$ & $x$ & $\checkmark$ & $\checkmark$ & $\checkmark$ & $\checkmark$ & $x$ & $x$ \\
\hline Paragios 2002 [49] & $\checkmark$ & $x$ & $\checkmark$ & $\checkmark$ & $\checkmark$ & $\checkmark$ & $x$ & $x$ \\
\hline Vazquez 2009 [62] & $\checkmark$ & $x$ & $\checkmark$ & $x$ & $x$ & $\bar{\checkmark}$ & $x$ & $x$ \\
\hline Ukwatta 2012 [60] & $\checkmark$ & $x$ & $x$ & $\checkmark^{\dagger}$ & $x$ & $\checkmark$ & $x$ & $\checkmark$ \\
\hline Rajchl 2012 [51] & $\checkmark$ & $\checkmark$ & $x$ & $x$ & $x$ & $\checkmark$ & $\checkmark$ & $\checkmark$ \\
\hline Delong 2009 [18] & $\checkmark$ & $\checkmark$ & $\checkmark^{*}$ & $\checkmark$ & $x$ & $x$ & $\checkmark$ & $\checkmark$ \\
\hline Ulén 2013 [61] & $\checkmark$ & $\checkmark$ & $\checkmark^{*}$ & $\checkmark$ & $x$ & $x$ & $\checkmark$ & $\checkmark$ \\
\hline Schmidt 2012 [56] & $\checkmark$ & $x$ & $\checkmark^{* *}$ & $\checkmark$ & $\checkmark$ & $x$ & $\checkmark$ & $x$ \\
\hline Nosrati 2013 [45] & $\checkmark$ & $\checkmark$ & $x$ & $\checkmark$ & $x$ & $\checkmark$ & $\checkmark$ & $\checkmark$ \\
\hline our work & $\checkmark$ & $\checkmark$ & $\checkmark$ & $\checkmark$ & $\checkmark$ & $\bar{\checkmark}$ & $\bar{\checkmark}$ & $x$ \\
\hline
\end{tabular}

$\circ$ Works for special cases only.

$\dagger$ The distance constraint is imposed after pre-segmenting the inner region.

\# Exclusion constraint is a non-submodular constraint and the specified methods cannot guarantee the global solution for such constraint.

* Changing the distance constraints requires reconstructing the graph. Further, increasing the thickness increases the storage memory requirements. In contrast, in our work we can change the thickness by simply changing a parameter, i.e. $d$ in (2) or $\left|\mathcal{C}_{i j}\right|$ in (6), and memory usage in our case is independent of thickness constraint.

** The attraction force between regions has not been discussed in [56] However, since they include containment similar to [18], it seems that they are able to enforce attraction between regions' boundaries.

functions has taken several forms : (i) adding new energy terms, e.g. edge, region, shape, statistical overlap and area prior terms [4], [6], [7], [9], [46], [50], [57], [69]; (ii) extending binary segmentation methods to multi-label segmentation [3], [23], [33], [63]; (iii) incorporating spatial relationships between labels, objects, or object regions [15], [19], [22], [31], [37], [53]; and (iv) automatically setting objective function parameters [2], [27], [38], [39], [47], [52], [59].

Here, we focus on augmenting the level set formulation to impose containment and exclusion constraints between different regions. We have chosen these two constraints due to their descriptive power in segmenting compound objects. The level set-based methods have been widely used in computer vision over the years and proven to be useful for medical image segmentation [5], [29], [41], [49] due to their several advantages such as 1) parametrization independence, 2) the ease of implementation, 3) their ability to deal with topological changes, 4) the ease of extendibility from a curve in 2D to higher dimensions (e.g. surfaces and hyper-surfaces) and 5) their ability to impose different image data and prior knowledge terms and control their contributions in segmentation tasks. However, we believe that level set has not reached its full potential yet. In the next section, we review related works that incorporate geometrical constraints into the segmentation formulation.

\section{A. Related works}

Encoding spatial relations between multiple target objects is not new. Some existing methods encode spatial relationships via relative inter-object distances, e.g. [30]. Other works incorporated fuzzy spatial relationships. For example, Colliot et al. [15] proposed direction and distance constraints into deformable models, whereas Gould et al. [22] learned relative super-pixel location priors for a probabilistic, conditional Markov random field (MRF) multi-class segmentation. In [37], the authors utilized interacting organisms in an artificial life framework to encode spatial relationships between objects. However, the above mentioned methods do not, for example, handle containment constraints.

Atlas-based segmentation has also been particularly useful in medical image analysis applications. The atlas has the ability to encode the (non-pathological) spatial relationships between multiple tissue types, anatomical structure or organs [14], [20]. However, this has so far been restricted to single (albeit multi-part or multi-region) object instance, i.e. it does not address spatially-recurring objects in the scene. Also, atlases usually are built from datasets of manually segmented images. These manual segmentations may not always be available, or it might not be straightforward to define a representative template for a given object (e.g. the nerve cells in Figure $6(f, g))$.

Over the past decade, much attention has been given to incorporating geometrical constraints into the segmentation objective function. In the continuous domain, Zheng et al. [70], Goldenberg et al. [21] and Paragios [49] proposed methods that are based on coupled surfaces propagation to segment a single object in an image. In [62], Vazquez et al. defined elastic coupling between multiple level set functions to model ribbonlike partitions. However, their approach was not designed to handle interactions between more than two regions. Wu et al. [67] also proposed a method for segmenting a region bounded by two coupled terrain-like surfaces by minimizing the intraclass variance. Their method, however, is limited to handling objects that can be "unfolded" into two coupled surfaces and it can segment only a single object in an image.

Ukwatta et al. [60] also proposed a method based on coupling two surfaces for Carotid adventitia (AB) and lumenintima (LIB) segmentation. The advantage of their work over previous works is that they optimized their energy functional by means of convex relaxation. However, their method was restricted to only segment objects with coupled surfaces. Using the same framework as [60], Rajchl et al. [51] presented a graphical model to segment the myocardium, blood cavities and scar tissue. Their method used seed points as hard constraints to distinguish the background from myocardium. They did not impose any thickness constraint between different surfaces, i.e. no attraction/repulsion forces between surfaces, and their method was only validated by segmenting a single (not multi-recurring) object. Recently, Nambakhsh et al. [42] proposed an efficient method for left ventricle (LV) segmentation by iteratively minimizing a convex upper bound energy functional for a coupled surface. Their method implicitly imposes a distance between two surfaces by learning the LV 
shape. They showed that their method only needs one fully segmented subject to train the LV shape.

In the discrete domain, a few recent works have focused on tiered segmentation to encode adjacency relationships such as "centre", "left", "right", "above" and "bottom" [19], [31]. These works were generalized by Strekalovskiy et al. in [58], but remain incapable of handling the geometrical containment constraint. Li et al. [28] proposed a method to segment nested objects by defining distance constraints between the object's surfaces with respect to a center point. Given that it was formulated using polar coordinates, their method could only handle star-shaped objects. Delong and Boykov [18] and Ulén et al. [61] encoded geometric interactions (including containment and exclusion) between distinct regions into a graph-cut framework. Both these methods have been proposed in the discrete domain and hence tend to exhibit a grid bias (metrication error) as well as large memory usage. Their methods guarantee the global solution for containment constraint. Schmidt et al. [56] modified [18] by adding the Hausdorff distance prior to the MRF-based segmentation framework to impose maximum distance constraint. Inspired by [18], [61], Nosrati et al. [45] proposed a method to encode containment and detachment, between different regions with a specified minimum distance between their boundaries in the continuous domain. Their approach guarantees the global optimal solution using functional lifting technique. However, their work cannot encode some constraints that have containment and exclusion at the same time, e.g. A contains $\mathrm{B}$ and $\mathrm{C}$ while $\mathrm{B}$ and $\mathrm{C}$ are excluded from one another.

\section{B. Contributions}

In this work, we focus on encoding two useful geometric constraints, containment and exclusion between the regions of multi-region objects, into the level set formulation. Using level sets embedding functions that are based on distance transforms (as is usually done) enables us to naturally enforce optional distance constraints between different regions. Our framework can enforce attraction forces as well as minimum and maximum distances between regions' boundaries. This work can be seen as a counterpart of the work of Delong and Boykov [18] and Ulén et al. [61] but in a continuous local optimization framework.

In graph-based methods, e.g. [18] and [61], increasing the distance (or thickness) between regions requires more edges be added to the underlying graph, which increases the memory usage and computation time. In contrast, in our work, changing the distance constraints is performed by simply changing a variable and the memory usage in our case is independent of distance constraint.

In addition, due to our continuous formulation, our method is free from metrication error, unlike graph-based methods that tend to exhibit a grid bias [43]. Metrication error is defined as the artifacts which appear in graph-based segmentation methods due to penalizing region boundaries only across axis aligned edges. Metrication error can be reduced in graphbased methods by increasing the graph connectivity that also increases memory usage even more.
We evaluate our framework on different biomedical applications and modalities including histology/microscopy images, cardiac MRI, brain positron emission tomography (PET) and blood vessel segmentation in lung CT. However, our comprehensive quantitative validation mainly focuses on two applications: histology and cardiac MRI analysis. Histology is crucial for studying diseases such as cancer and for obtaining reference diagnosis (e.g. biopsy histopathology). Cell segmentation is an important step for automating histological and microscopic image analysis, e.g. for disease diagnosis. Objects in histological microscopy images, such as cellular organization of tissue, typically exhibit multiple evident spatial relationships like containment and exclusion. The ability of level setbased methods to automatically handle topological changes makes them suitable for segmenting spatially-recurring multiregion cells, while satisfying the above mentioned constraints. Also, due to our local optimization, our method requires less memory compared to global graph-based approaches. In practice, memory usage becomes important when we deal with very large microscopy images and $3 \mathrm{D}$ volumes.

We also evaluate our method on publicly available cardiac datasets, where we segment the endo- and epicardium of the left and right ventricles simultaneously in 3D MRI and compare our results with state-of-the art methods.

It is worth mentioning that despite all advantages of level set-based method, due to the local optimization framework, the final segmentation results will depend on the initialization and hence it is important to take this into account for a successful segmentation, whereas results in global methods, e.g. [18], do not depend on initialization. Nevertheless, as seen Section V, we obtained encouraging results even with fully automatic or rough initialization that is distant from the desired boundaries. Table I compares certain features of our work with popular and state-of-the-art methods.

The paper is organized as follows: Section II describes our constraints and geometric interaction terms for segmenting a two-region object. The extension to multi-region is described in Section III. Section IV details the optimization procedure. Section V presents our experimental validation and evaluation in different applications. Following a note and example results of a special case of distance constraints in Section VI, we conclude in Section VII.

\section{PROBLEM FORMULATION}

We first introduce the concepts of containment and exclusion, the two intuitive geometrical constraints, we use in this work to segment an image with two-region objects. Containment: We say region $\mathrm{A}$ contains region $\mathrm{B}$ if $\mathrm{B}$ is completely encapsulated by A, i.e. $A \cap B=B$. We also add an optional distance constraint to this term; allowing us to specify that $\mathrm{B}$ is inside $\mathrm{A}$ with a distance of $d$ pixels between their boundaries, perhaps with an attraction/repulsion force between their boundaries. Exclusion: We say region A and $\mathrm{B}$ are excluded from one another if they are disjoint, i.e. $A \cap B=\emptyset$. We also add an optional distance constraint to this term; allowing us to specify that $\mathrm{A}$ and $\mathrm{B}$ are disjoint with a minimum distance of $d$ pixels between their boundaries. 


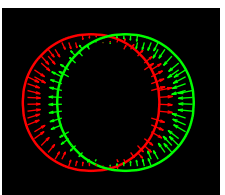

(a)
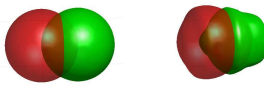

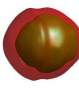

(b)
Figure 1: Containment constraint between two red and green regions. (a) Attraction and repulsion applied to the red and green boundaries when the red region contains the green one. (b) Surface (zero level set) evolution (from left to right): red contains green with a predefined distance between them.

For clarity, we first formulate the containment and exclusion energy terms for two-region objects (surrounded by the background).

\section{A. Containment energy}

Let $\Omega$ be a bounded open subset of $\mathbb{R}^{n}$ where $n$ is the image dimension (in this work $n=2$ or 3 ) and $I: \Omega \rightarrow \mathbb{R}$ is a given image (scalar field) $)^{1}$. In our formulation, $C_{i}$ represents the boundary (or surface) of the $i^{\text {th }}$ region in a multi-region object. We also define $\phi_{i}(x): \Omega \rightarrow \mathbb{R}$ as the signed distance function corresponding to $C_{i}$, where $\phi_{i}(x)>0$ is inside and $\phi_{i}(x)<0$ is outside the region $i . C_{i}$ and $\phi_{i}$ are related by the zero level set of $\phi_{i}$; i.e., $C_{i}=\left\{x \mid \phi_{i}(x)=0\right\}$. Using the signed distance functions enables us to efficiently control the relative distance $d$ between the objects surfaces. Given the two regions $i$ and $j$, our containment energy, such that $i$ contains $j$ with a distance of $d$ pixels between their surfaces is

$$
E_{C}\left(\phi_{i}, \phi_{j} ; d\right)=\int_{\Omega}\left\|\phi_{i}(x)-\phi_{j}(x)-d\right\|^{2} d x .
$$

If $d=0$, minimizing $E_{C}$ encourages $\phi_{i}$ and $\phi_{j}$ to be identical. Adding a positive or negative constant $d$ to a signed distance function $\phi$, dilates and shrinks the region specified by $\phi$, respectively. To better illustrate (1), we re-write (1) as $\int_{\Omega}\left\|\left(\phi_{i}(x)-d\right)-\phi_{j}(x)\right\|^{2} d x$ that penalizes the difference between $\phi_{j}(x)$ and the shrinked $\phi_{i}$, i.e., $\phi_{i}(x)-d$. This causes the surface $i$ to contain $j$ while maintaining the distance of $d$ pixels between them. $E_{C}$ in (1) creates attraction and repulsion between $i$ and $j$ surfaces, by penalizing the area in which $\phi_{i}$ and $\phi_{j}$ are different.

Figure 1(a) shows the attraction and repulsion along the boundaries of $i$ and $j$ when $i$ is encouraged to contain $j$. Figure 1(b) shows how the two surfaces $i$ and $j$ attract each other while maintaining the predefined distance $d$ between them by solving the Euler-Lagrange equation for (1):

$$
\frac{\partial \phi_{i}}{\partial t}=-2\left(\phi_{i}-\phi_{j}-d\right), \quad \frac{\partial \phi_{j}}{\partial t}=2\left(\phi_{i}-\phi_{j}-d\right),
$$

where $t$ is an artificial time variable.

\section{B. Exclusion energy}

In many segmentation applications, there might be a need to exclude objects from one another; e.g., we might be interested

\footnotetext{
${ }^{1}$ Our method extends directly to non scalar fields, e.g. color images, vector fields, or tensor fields, by modifying the data terms, as in [12], [64]
}

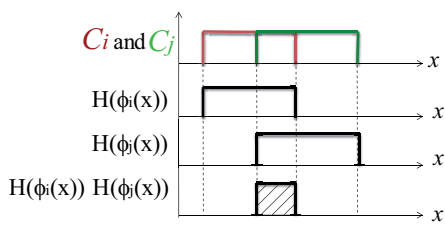

(a)

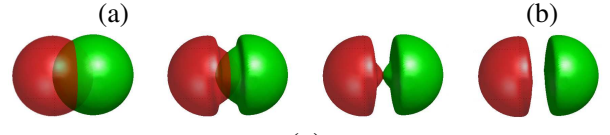

(c)

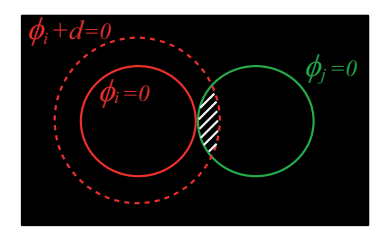

(b)

Figure 2: Exclusion ( $i$ excludes $j$ ): (a) Shared area between regions $i$ and $j$ (the shaded area) is penalized by (3). (b) Adding a positive constant $d$ to $\phi_{i}$ dilates region $i$ and penalizes any region $j$ that is within distance $d$ pixels from $i$ (shaded area). (c) Surface evolution (from left to right): Green and red are excluded from one another with a predefined distance between them.

in segmenting the regions that reside outside another region. Following Zhao et al. [71], we enforce an exclusion constraint on two regions by penalizing the area that the two regions share. The proposed energy term for excluding regions $i$ and $j$ from one another is

$$
E_{E}\left(\phi_{i}, \phi_{j}\right)=\int_{\Omega} H\left(\phi_{i}(x)\right) H\left(\phi_{j}(x)\right) d x,
$$

where $H($.$) is the regularized Heaviside function. Figure 2(a)$ shows how the shared area between two regions is penalized (without any distance between the regions). Similar to (1), the distance condition between two surfaces can be easily added for the exclusion term as well. The distance $d$ can be added to either $\phi_{i}$ or $\phi_{j}$ in (3). Adding a positive constant value $d$ to $\phi_{i}$ dilates region $i$ and ensures that any region $j$ within the distance of $d$ pixels from $i$ is penalized (white shaded area in Figure 2(b)). Similarly if $d>0$ is added to $\phi_{j}, \phi_{i}$ will be pushed away from $j$ by $d$ pixels. Note that adding $d$ to both $\phi_{i}$ and $\phi_{j}$ causes a final distance of $2 d$ pixels between the two surfaces. Figure 2(c) shows a 3-D evolution of surfaces $i$ and $j$ by solving the Euler-Lagrange equation for (3):

$$
\frac{\partial \phi_{i}}{\partial t}=-\delta\left(\phi_{i}\right) H\left(\phi_{j}\right), \quad \frac{\partial \phi_{j}}{\partial t}=-\delta\left(\phi_{j}\right) H\left(\phi_{i}\right),
$$

where $\delta(z)=d H(z) / d z$ is the regularized Dirac delta function.

\section{Regional and regularization terms}

The above mentioned energy terms control the geometrical interactions between regions of an object and are independent of image data. Assuming piecewise constant regional intensities and adopting the total variation regularization, we employ the standard Chan-Vese formulation [13] to minimize the intraregion variance considering the geometric, e.g. containment, between regions. For a three-region $(i, j$ and background) 
scenario in which $i$ contains $j$, the regional term is:

$$
\begin{aligned}
E_{I}\left(\phi_{i}, \phi_{j} ; g_{i}, g_{j}, g_{b g}\right)= & \int_{\Omega}\left(g_{j}(x) H\left(\phi_{j}(x)\right)\right. \\
& +g_{i}(x) H\left(\phi_{i}(x)\right) H\left(-\phi_{j}(x)\right) \\
& \left.+g_{b g}(x) H\left(-\phi_{i}(x)\right)\right) d x \\
& +\int_{\Omega}\left|\nabla H\left(\phi_{i}(x)\right)\right| d x \\
& +\int_{\Omega}\left|\nabla H\left(\phi_{j}(x)\right)\right| d x
\end{aligned}
$$

where $g_{i}=\left|I(x)-\mu_{i}\right|^{2}, \mu_{i}$ is the intensity prior for region $i$ and $H\left(\phi_{i}\right)$ and $H\left(-\phi_{i}\right)$ indicate the inside and outside region $i$, respectively. The last two terms in (5) are the regularization terms that smooth the surfaces $i$ and $j$ by penalizing their surface area.

So far, we introduced our constraints for segmenting an image with two-region objects and background. In the next section, we extend our framework to segment objects with more than two-regions.

\section{EXTENSION TO MULTI-REGION OBJECTS}

To extend our framework to multi-region objects segmentation, we introduce an $R \times R$ constraints matrix, $\mathcal{C}$, that encodes the containment and exclusion constraints, where $R$ is the number of regions of an object in the image to be segmented. Our motivation to introduce $\mathcal{C}$ is to provide a framework where one can encode geometrical constraints (containment/exclusion) easily and intuitively. Table II shows how we encode containment and exclusion constraints into matrix $\mathcal{C}$.

Table II: Encoding containment and exclusion into matrix $\mathcal{C}$

\begin{tabular}{|c|c|}
\hline Constraint & Matrix $\mathcal{C}$ \\
\hline$i$ contains $j$ & $\mathcal{C}_{i j}>0$ \\
$i$ and $j$ are disjoint & $\mathcal{C}_{i j}<0$ AND $\mathcal{C}_{j i}<0$ \\
$i$ and $j$ have no constraint & $\mathcal{C}_{i j}=\mathcal{C}_{j i}=0$ \\
\hline
\end{tabular}

In addition to encoding the containment and exclusion constraints, we also encode the distance constraint into $\mathcal{C}$ such that $S_{i j}=\operatorname{sign}\left(\mathcal{C}_{i j}\right)$ defines the containment or exclusion of objects $i$ and $j$ ( $S_{i j}>0$ means $i$ contains $j$ and $S_{i j}=S_{j i}<0$ means $i$ and $j$ are excluded from one another), and $\left|\mathcal{C}_{i j}\right|$ indicates the distance between these two regions. Note that $\operatorname{sign}(\mathcal{C})$ is symmetric with respect to exclusion, i.e., if $i$ excludes $j, j$ also excludes $i$.

We extend $E_{C}$ in (1) to more than two regions by identifying all positive entries in $\mathcal{C}$ (i.e. containment) as follows:

$$
\begin{aligned}
& E_{C}^{\text {total }}(\boldsymbol{\Phi} ; \mathcal{C})= \\
& \sum_{i=1}^{R} \int_{\Omega}\left(\prod_{j \mid \mathcal{C}_{i j}>0}\left\|\phi_{i}(x)-\phi_{j}(x)-\mathcal{C}_{i j}\right\|^{2}\right) d x
\end{aligned}
$$

where $\boldsymbol{\Phi}=\left\{\phi_{1}, \ldots \phi_{R}\right\}$.

Exclusion energy can also be extended to multi-region objects by adding an exclusion term (as describes in (3)) for any pair of objects that must adhere to an exclusion constraint. Given matrix $\mathcal{C}$, the total exclusion energy is:

$$
\begin{aligned}
& E_{E}^{\text {total }}(\boldsymbol{\Phi} ; \mathcal{C})= \\
& \sum_{i=1}^{R} \sum_{j>i}^{R} \frac{S_{i j}\left(S_{i j}-1\right)}{2} \int_{\Omega} H\left(\phi_{i}-\mathcal{C}_{i j}\right) H\left(\phi_{j}-\mathcal{C}_{j i}\right) d x .
\end{aligned}
$$

where the term $\frac{S_{i j}\left(S_{i j}-1\right)}{2}$ is equal to one, only if $\mathcal{C}_{i j}=\mathcal{C}_{j i}<$ 0 , i.e., $i$ excludes $j$, and it is zero otherwise. Since matrix $\mathcal{C}$ is symmetric with respect to exclusion, it is sufficient to incorporate only the upper triangle of $\mathcal{C}$ in (8).

Finally, to extend the regional energy term and to simplify the formulation, we note that regions not contained by any other region are contained by the background. So we add a dummy background row (say row 0 ) to matrix $\mathcal{C}$ that is positive in the columns that do not have any positive value (i.e. are not contained by other regions). Recalling that $g_{i}=\left|I-\mu_{i}\right|^{2}$ and defining $\mathbf{g}=\left\{g_{0}, g_{1}, \ldots, g_{R}\right\}$, we extend (5) to multi-region by searching each row of $\mathcal{C}$ for positive values (containments) as follows:

$$
\begin{aligned}
E_{I}^{\text {total }}(\mathbf{\Phi} ; \mathcal{C}, \mathbf{g})= & \sum_{i=0}^{R} \int_{\Omega}\left(g_{i} H\left(\phi_{i}(x)\right) \prod_{j \mid \mathcal{C}_{i j}>0} H\left(-\phi_{j}(x)\right)\right) d x \\
& +\sum_{i=1}^{R} \int_{\Omega}\left|\nabla H\left(\phi_{i}(x)\right)\right| d x
\end{aligned}
$$

where $g_{0}=g_{b g}$ and $\phi_{0}=1$. The first and second terms in (9) are the multi-region extension of the regional and regularization terms in (5), respectively.

Combining (7-9), the total energy functional becomes:

$$
E_{\text {total }}=\lambda_{1} E_{I}^{\text {total }}+\lambda_{2} E_{C}^{\text {total }}+\lambda_{3} E_{E}^{\text {total }},
$$

where the positive constants $\lambda_{1}, \lambda_{2}$ and $\lambda_{3}$ control the contribution of each term in the segmentation.

\section{OPTIMIZATION}

To minimize the functional in (10), we follow the approach of Chan and Vese [13] and derive the Euler-Lagrange equation. The objective function (10) is a weighted sum of non-negative terms and it will become zero if and only if all its terms are zero. To minimize $E_{C}^{\text {total }}$ in (7), note that a region, represented by $\phi_{\ell}$, can contain other regions and can be contained by other ones (i.e., $\phi_{\ell}$ can appear as either $\phi_{i}=\phi_{\ell}$ or $\phi_{j}=\phi_{\ell}$ in (7)). For region $\phi_{\ell}$, the Euler-Lagrange equation associated to $E_{C}^{t o t a l}$ in (7) is calculated as:

$$
\begin{aligned}
& \sum_{\substack{j_{\ell} \mathcal{C}_{\ell j}>0 \\
F_{\ell}}}^{C} 2\left(\phi_{\ell}-\phi_{j}-\mathcal{C}_{\ell j}\right) \prod_{k \neq j \mid \mathcal{C}_{\ell k}>0}\left\|\phi_{\ell}(x)-\phi_{k}(x)-\mathcal{C}_{\ell k}\right\|^{2} \\
& -\sum_{i=1}^{R} S_{i \ell}\left(S_{i \ell}+1\right)( \\
& \left.\quad\left(\phi_{i}-\phi_{\ell}-\mathcal{C}_{i \ell}\right) \prod_{j \neq \ell \mid \mathcal{C}_{i j}>0}\left\|\phi_{i}(x)-\phi_{j}(x)-\mathcal{C}_{i j}\right\|^{2}\right)=0
\end{aligned}
$$


where the term $\frac{S_{i \ell}\left(S_{i \ell}+1\right)}{2}$ is equal to one, only if $\mathcal{C}_{i \ell}>0$, i.e., $\ell$ is contained by $i$, and is zero otherwise.

Deriving the Euler-Lagrange for $E_{E}^{\text {total }}$ in (8) for the level set $\phi_{\ell}$ results in the following equation:

$$
F_{\ell}^{E}=\sum_{i=1}^{R} \frac{S_{\ell i}\left(1-S_{\ell i}\right)}{2} \delta\left(\phi_{\ell}-\mathcal{C}_{\ell i}\right) H\left(\phi_{i}-\mathcal{C}_{i \ell}\right)=0 .
$$

To minimize $E_{I}^{\text {total }}$ in (9) for a specific level set, $\phi_{\ell}$, it should be noted that $\phi_{\ell}$ can appear in the product term in (9) (like $\phi_{j}$ in (9)) when $\mathcal{C}_{i \ell}>0$, i.e., $\frac{S_{i \ell}\left(S_{i \ell}+1\right)}{2}=1$. For the level set $\phi_{\ell}$, the Euler-Lagrange equation associated to (9) is:

$$
\begin{aligned}
& F_{\ell}^{I}=g_{\ell} \delta\left(\phi_{\ell}\right) \prod_{j \mid \mathcal{C}_{\ell j}>0} H\left(-\phi_{j}\right) \\
& -\sum_{i=0}^{R} \frac{S_{i \ell}\left(S_{i \ell}+1\right)}{2}\left(g_{i} H\left(\phi_{i}\right) \delta\left(\phi_{\ell}\right) \prod_{j \neq \ell, \mathcal{C}_{i j}>0} H\left(-\phi_{j}\right)\right) \\
& -\operatorname{div}\left(\frac{\nabla \phi_{\ell}}{\left|\nabla \phi_{\ell}\right|}\right)=0 .
\end{aligned}
$$

Having (11), (12) and (13), the update equation of region $\phi_{\ell}$ is calculated as:

$$
\frac{\partial \phi_{\ell}}{\partial t}=-\left(\lambda_{1} F_{\ell}^{I}+\lambda_{2} F_{\ell}^{C}+\lambda_{3} F_{\ell}^{E}\right) .
$$

Finally, in implementing the proposed method the level sets $\phi$ should remain signed distance maps during the optimization procedure, otherwise the distance priors do not work properly. To do so, the level sets functions are re-initialized to signed distance functions after every few $(\sim 5)$ iterations.

To ensure that our level set-based framework is numerically stable, we place an upper bound for the time-step $\partial t$, using the Courant-Friedrichs-Lewy (CFL) condition [17]. The stability condition is

$$
F_{\max } \partial t \leq \min \left(h_{x}, h_{y}, h_{z}\right),
$$

where $h_{x}, h_{y}$ and $h_{z}$ are the grid spacing in the $x, y$ and $z$ direction, respectively, and $F_{\max }$ is the maximum absolute force (also known as speed function) applied to the level set at each iteration and is calculated from (11),(12),(13). For $h_{x}=$ $h_{y}=h_{z}=1$, at each iteration we make sure that $\partial t \leq$ $1 / F_{\max }$. Violating the CFL condition results in instabilities.

\section{EXPERIMENTS}

In this section, we present several experiments and show the applicability and utility of our framework on different biomedical applications. However, our comprehensive quantitative validation mainly focuses on two important medical applications: (a) histology and microscopy image segmentation and (b) left and right cardiac ventricles segmentation. We also compare our method to the analogous discrete works of Delong and Boykov (DB) [18] and Ulén et al. (USK) [61] on both synthetic and real data and analyze the metrication error, running time and memory usage.

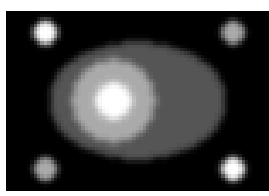

(a) Original image

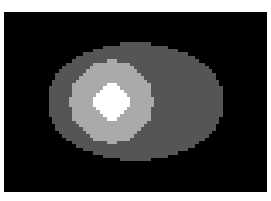

(d) GC: 8- connected

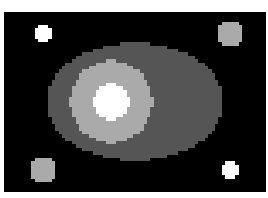

(b) ACWOE

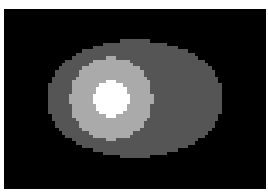

(e) GC: 16- connected

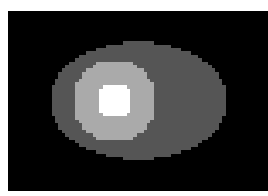

(c) GC: 4- connected

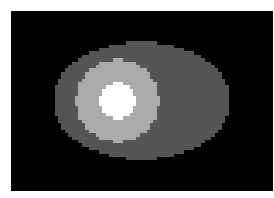

(f) Our method

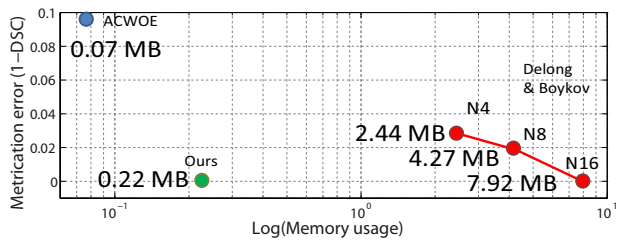

(g) Metrication error vs. memory usage

Figure 3: Synthetic three-region object segmentation. (b) ACWOE's result. (c-e) DB graph cuts based method [18] with different connectivities. (f) Our segmentation results. (g) Metrication error vs. memory usage: red curve: GC-based method; blue circle: ACWOE; green circle: our method.

\section{A. Synthetic data}

In our first experiment, we compare our method with DB in terms of metrication error and memory usage on a simple synthetic example. Metrication error is defined as the artifacts which appear in graph-based segmentation methods due to penalizing region boundaries only across axis aligned edges.

In Figure 3(a), we are interested in segmenting the 3-region elliptic object. Figure 3(b) shows a segmentation without imposing any geometrical constraint (here we used multiphase active contours without edges (ACWOE) [63]. DB's results for 4, 8 and 16 graph connectivity are shown in Figures 3(c-e). Note the metrication artifacts in (c) and (d).

In this experiment, we quantify the metrication error by metrication error $=\left(\mathrm{DSC}_{c}-\mathrm{DSC}_{d}\right)$, where $\mathrm{DSC}_{c}$ and $\mathrm{DSC}_{d}$ are Dice similarity coefficient for continuous and discrete methods, respectively. The DSC measures the segmented regions overlap and is given by $2|A \cap B| /(|A|+|B|)$, where $A$ and $B$ are the ground truth and the segmentation result regions, respectively. Since we used the same data term for DB and our method, and since the DSC of our continuous method is one for this synthetic data $\left(\mathrm{DSC}_{c}=1\right)$, the only source of error in DB's work is due to the gridding bias. The metrication error in graph based methods can be reduced by increasing the graph connectivity at the expense of increasing the memory usage.

Memory usage of our method and the graph-based methods are compared in Figure $3(\mathrm{~g})$. The red curve in Figure $3(\mathrm{~g})$ illustrates the metrication error vs. memory usage of DB for 4,8 and 16 connectivity, while the blue and green circles represent ACWOE and our method, respectively. To remove the metrication error, DB needed 16 connectivity, Figure 3(e), requiring 36 times more memory than our method (7.92 MB 


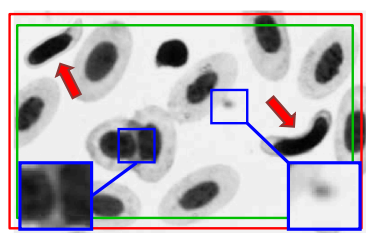

(a)

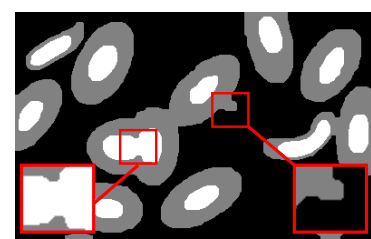

(b)

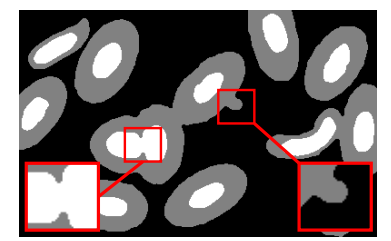

(c)

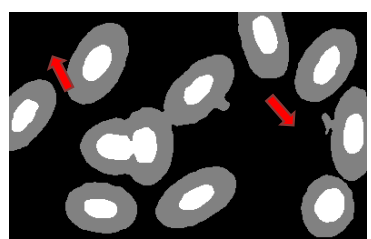

(d)

Figure 4: Cell segmentation in a microscopy image. (a) Original image, $250 \times 395$ pixels. Arrows show abnormal cells. (b) Result of DB, 33.90 MB. (c) Our result (thickness= 2 pixels, $1.50 \mathrm{MB}$ ). (d) Our result when segmenting only normal looking (elliptical) cells (thickness=10 pixels, $1.50 \mathrm{MB}$ ). Note that DB needs $313.41 \mathrm{MB}$ extra memory (347.31 MB in total) to impose a thickness constraint of 10 pixels while the memory usage of our method is independent of thickness constraint.

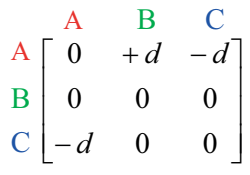

(a) $\mathcal{C}$ matrix

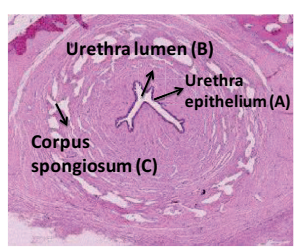

(b) Original image

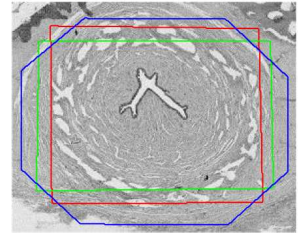

(c) Initialization

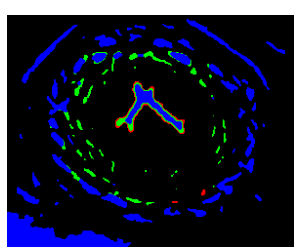

(d) ACWOE

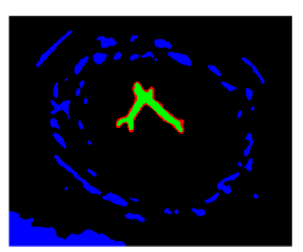

(e) Our method

Figure 5: Urethra segmentation in a histology image. The constraint matrix is set such that the urethra epithelium (A) contains the urethra lumen (B) and excludes the other regions with similar intensity with B, i.e. the corpus spongiosum (C). Here A, $\mathrm{B}$ and $\mathrm{C}$ are represented by red, green and blue colors, respectively.

vs. $0.22 \mathrm{MB}$ ). We emphasize that DB needs more memory largely due to exploring the whole search space to find the global solution. The larger the size of the image (e.g. higher resolution $2 \mathrm{D}$ microscopy images that can be in the order of 100 s of mega-pixels or 3D volumes), the more important it is to pay attention to this increased memory consumption. In this work, we sacrificed the optimality (i.e. via our local optimization with level sets) for memory efficiency.

\section{B. 2D histological and microscopic images}

Histology and microscopy image analysis is becoming increasingly crucial for studying diseases such as cancer and for obtaining reference diagnoses. Two important features that can be seen frequently in many histology and microscopy images are (a) the existence of multiple objects of the same class (e.g. cells) in a single image, and (b) the geometric interactions between the objects' regions. The latter includes containment and exclusion between the objects and different parts of the objects (e.g. a cell and its sub-cellular components, such as nucleus and nucleolus).

Figure 4 compares our method with DB in fish blood cell segmentation. Figures 4(b) and (c) show DB's and our results, respectively. Note the gridding artifacts in DB's work (red boxes). In both ours and DB's work, we can adjust the distance (thickness) between different regions to control which objects are segmented. To exclude the abnormal cells (red arrows in Figure 4(a)) from the segmentation, we increase the imposed thickness constraint between two regions from 2 pixels to 10 pixels, Figure 4(d). In DB, increasing the thickness requires graph reconstruction with additional edges. The new graph needs an extra $\sim 313.41$ MB memory, an almost 10 fold increase. In contrast, we emphasize that in our method, thickness can be increased by simply changing the value of $d$ in (1) or equivalently $\left|\mathcal{C}_{i j}\right|$ in (6) and it does not affect the memory usage.

Figure 5(a) shows how the containment and exclusion constraints are encoded into matrix $\mathcal{C}$ to segment and distinguish the urethra from other regions with similar intensity/color in a histological image of the ureter (Figure 5(b)). Note how our method is able to distinguish the urethra from other similar regions by forcing it to be contained in the urethral epithelium (Figure 5(e)), while the conventional active contours without edges (ACWOE), or any other method without containment and exclusion constraint, is incapable of distinguishing the urethra from other similar regions based solely on image pixel values (Figure 5(d)).

We tested our algorithm on 20 histology and microscopy images and used the DSC to evaluate the performance of our method. Figure 6 presents qualitative results on histology and microscopy data. The first and the second rows in Figure 6 show the original image and initialization overlaid on the original image. The third row shows multi-phase ACWOE results. The fourth and fifth rows show USK results with 4-connected and 8-connected graphs, respectively, and the bottom row shows the proposed method's results. These results illustrate the importance of geometrical constraints in histology/microscopy image segmentation and also show the effects of metrication error ( $4^{t h}$ and $5^{t h}$ rows). We note that we tried to get the best results as we could for USK's method by exhaustively searching for the best regularization weight and thickness (minimum distance) constraint. As is seen in Figure 6 , the metrication error in the 8 -connected graph $\left(5^{\text {th }}\right.$ row) is improved compared to the 4 -connected graph ( $4^{\text {th }}$ row) but not completely resolved. Penalizing boundaries of objects only across axis aligned edges in graph-based methods makes it difficult for a convex regularizer like total variation (TV) to be implemented in the discrete domain efficiently. Boykov 
(a)
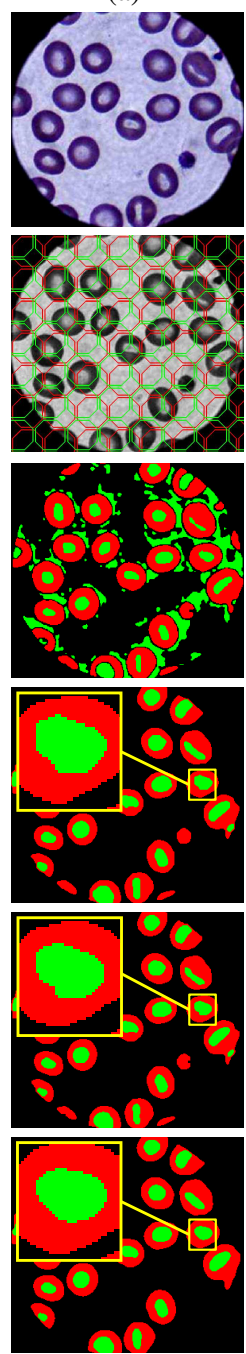

(b)
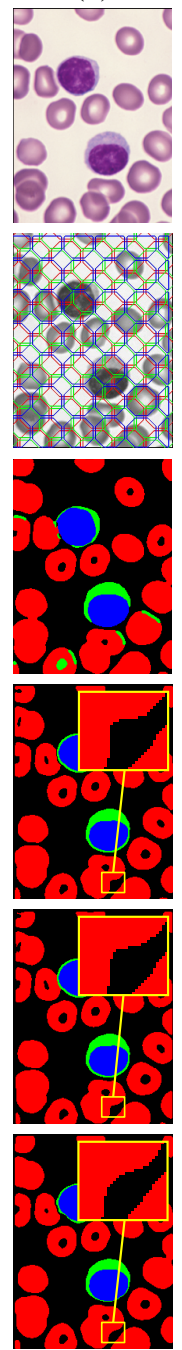

(c)
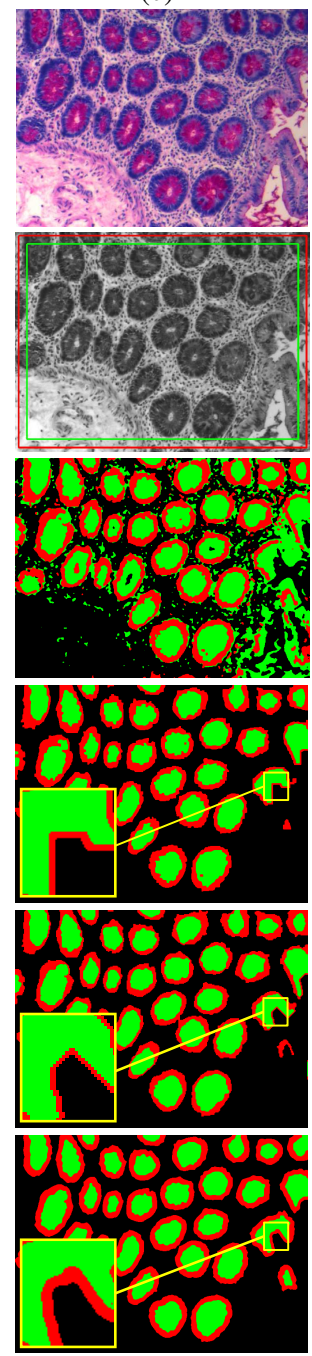

(d)
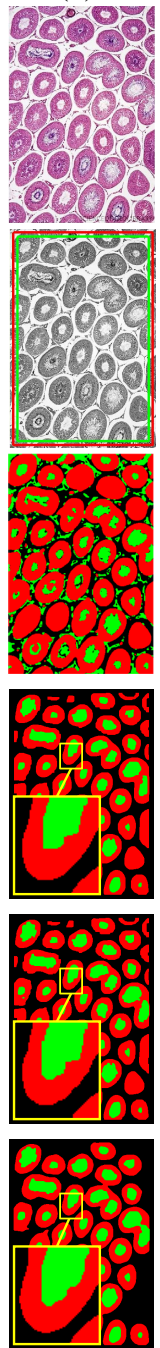

(e)
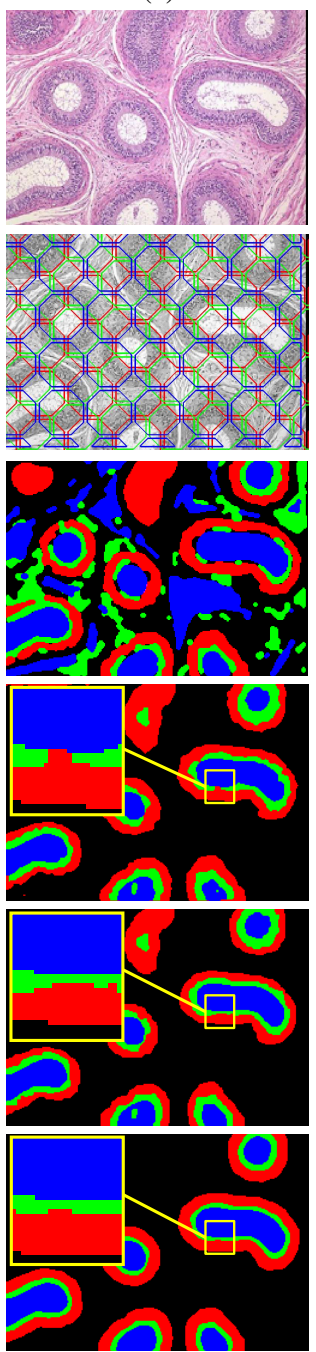

(f)
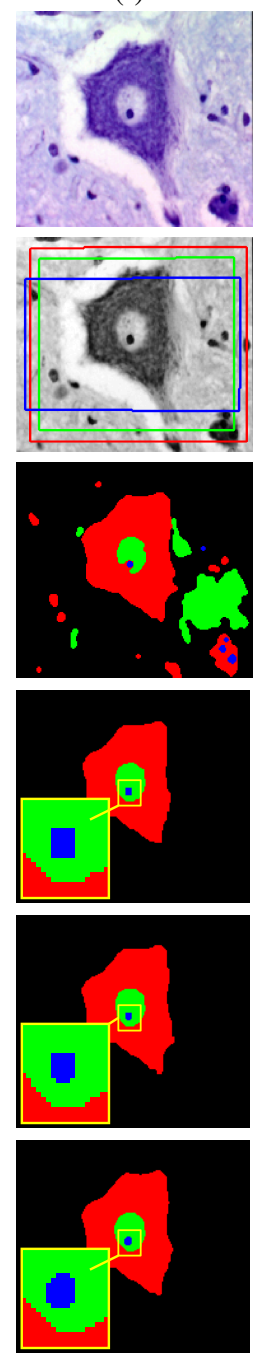

(g)
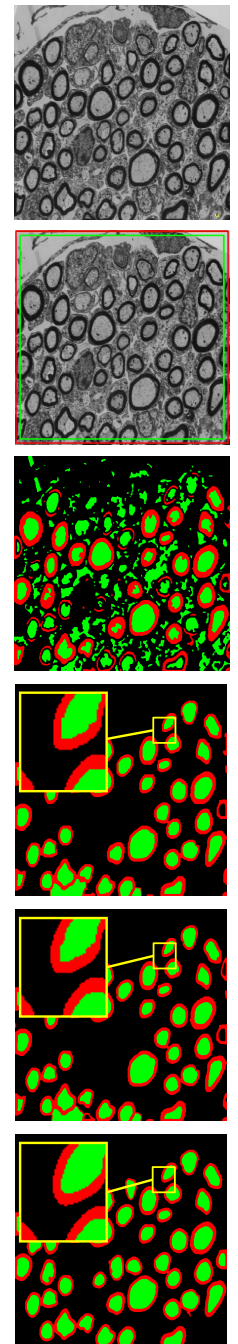

Figure 6: Segmentation of histology and microscopy images. $1^{\text {st }}$ row: Original image. $2^{\text {nd }}$ row: Initialization. $3^{\text {rd }}$ row: ACWOE results. $4^{\text {th }}$ row: USK's method with 4-connectivity. $5^{\text {th }}$ row: USK's method with 8 -connectivity. $6^{\text {th }}$ row: Our results. The images from left to right are: (a,b) Microscopy images of blood cells, (c,d,f) histological cross sections of testes histology and $(\mathrm{e}, \mathrm{g})$ neuron histology. Note that in case of no exclusion constraint USK is equivalent to DB.

and Kolmogorov [8] proposed a method to roughly overcome this issue. However, their method requires extra memory and computational time due to adding extra edges to the graph. On the other hand, the continuous frameworks, including level set framework, can efficiently encode general convex regularizers like TV.

We emphasize that in this experiment there was no need to initialize too close to the solution and we performed fully automatic initialization for almost all of the cases, Figure 6(a$\mathrm{e}, \mathrm{g})$. Indeed, using the regions close to the image boundary was sufficient for initialization. However, to show the effect of the initialization on the results, we ran our algorithm starting from three different initializations (Figure 7). Note that the obtained results from the first two initializations, Figure $7(\mathrm{a}, \mathrm{b})$, are almost identical, despite significant difference in their initialization. Yet the third result is affected by the initialization, Figure 7(c).

We evaluated our segmentation method by measuring the overlap between segmented and ground truth regions using
DSC. We report the DSC for contained regions and notcontained regions separately in Table III. As expected, our results for contained regions improved dramatically over the conventional ACWOE as the latter only considers the image intensity/color for its external energy term. In many histology and microscopy images, regions of interest and the background might have similar intensities making ACWOE insufficient for this task. For the same reason, other methods that do not enforce such constraints are not able to segment the contained regions properly. For the regions that have not been contained by other regions, both methods have a similar accuracy. However, our method indirectly improves the performance for these regions as well due to the attraction and repulsion created by the containment energy terms. The average memory usage to segment this microscopic histology dataset is $120 \mathrm{MB}$ and 1.86 MB for USK (4-connected graph) and our method, respectively.

Comparing the computation time of our method with a global graph based method, e.g. DB, is critical as we use 

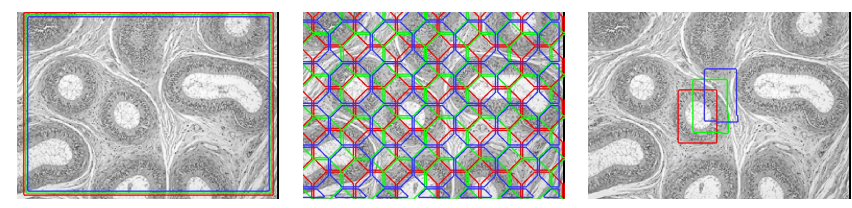

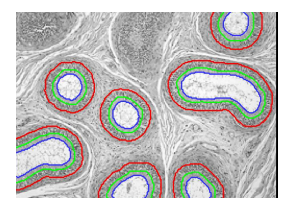

(a)

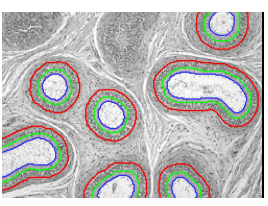

(b)

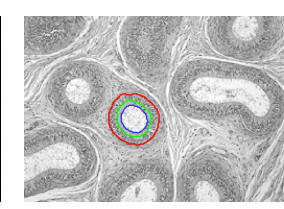

(c)
Figure 7: Effect of initialization on the results. Top row: initializations. Bottom row: results after convergence. Despite significant different initializations in (a) and (b), the obtained results are almost identical. Yet the third result (c) is clearly affected by the initialization.

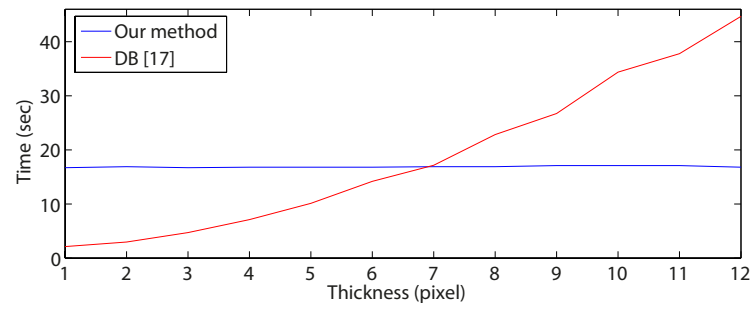

Figure 8: CPU runtime versus thickness/distance constraint.

local optimization and convergence time depends on how close we initialize the contours. In addition, the computation time for graph based methods, e.g. DB, highly depends on the thickness/distance constraint and largely varies from one image to another. To have a fair run time comparison, we created a $500 \times 500$ synthetic image consisting of several two-region nested objects with different distance between their regions. To initialize our method, we place the initial contours at the border of the image, e.g. similar to Figure $6(\mathrm{~d}, \mathrm{~g})$. Figure 8 compares the runtime between our method and DB for different thickness constraints. To impose a distance constraint of $T$ pixels between two regions, DB and USK need to add $O\left(T^{2}\right)$ extra edges per pixel. Therefore, these graph based methods are highly efficient in segmenting images with reasonable size and thickness constraint. On the other hand, for large distance constraints DB and USK are not that efficient (considering that they still provide us with the global solution) while in our framework the runtime is almost constant with respect to different distance constraints.

\section{Cardiac ventricles segmentation}

We also evaluated our framework on left and right cardiac ventricles segmentation. To this end, we used two different 3D datasets: 1) The Sunnybrook Health Science Centre dataset for left ventricle segmentation used in the MICCAI 2009 challenge [11], and 2) The Rouen University Hospital data for right ventricle segmentation used in the MICCAI 2012 challenge [24].
Table III: DSC and memory usage comparison

\begin{tabular}{|c|c|c|c|}
\hline & ACWOE [63] & USK [61] & Our method \\
\hline $\begin{array}{l}\text { Regions that } \\
\text { have not been } \\
\text { contained by } \\
\text { other regions }\end{array}$ & $0.88 \pm 0.05$ & $\begin{array}{l}\text { 4-C: } 0.89 \pm 0.04 \\
\text { 8-C: } 0.90 \pm 0.05\end{array}$ & $0.91 \pm 0.02$ \\
\hline $\begin{array}{c}\text { Regions that } \\
\text { contained by (or } \\
\text { excluded from) } \\
\text { others }\end{array}$ & $0.54 \pm 0.14$ & $\begin{array}{l}\text { 4-C: } 0.89 \pm 0.05 \\
\text { 8-C: } 0.90 \pm 0.04\end{array}$ & $0.90 \pm 0.04$ \\
\hline \multirow[t]{2}{*}{ Overall } & $0.68 \pm 0.07$ & $\begin{array}{l}\text { 4-C: } 0.89 \pm 0.04 \\
\text { 8-C: } 0.90 \pm 0.05\end{array}$ & $0.91 \pm 0.03$ \\
\hline & $\begin{array}{c}\text { Memory usage } \\
\text { (MB) }\end{array}$ & $\begin{array}{l}\text { 4-C: } 120 \pm 99.30 \\
\text { 8-C: } 167 \pm 101.60\end{array}$ & $1.86 \pm 1.37$ \\
\hline
\end{tabular}

1) Left ventricle segmentation: Left ventricle (LV) segmentation is an important step for the diagnosis of cardiovascular diseases. Accurate calculation of key clinical parameters such as ejection fraction, myocardium mass, and stroke volume depends on accurate segmentation of endocardial and epicardial boundaries of the left ventricle. We used our framework to segment the left ventricle. To model the LV, we encode the constraint "myocardium contains the left ventricle" into our framework.

We evaluated our method on the Sunnybrook Health Science Centre dataset. This dataset consists of 30 short-axis cardiac cine-MR images (15 volumes for training and 15 volumes for validation) obtained by a $1.5 \mathrm{~T}$ GE Signa MRI. All the images were obtained during 10-15 second one breath-hold with a temporal resolution of 20 cardiac phases over the heart cycle ${ }^{2}$.

The ground truth of endocardial and epicardial contours have been provided by an experienced cardiologist in all slices at end-diastole (ED) and end-systole (ES) phases.

Figure 9 shows the result for one subject over different slice levels: basal, mid-cavity and apical. The red and green curves indicate the epi- and endocardial boundaries, respectively. The distance between the epi- and endocardial boundaries (myocardium thickness) is not fixed but decreases from basal to apical level. Hence, we cannot choose a fixed distance prior $d$ for LV segmentation (c.f. (1)). Instead, we allow $d$ to vary linearly from the first slice, at the basal level, to the last slice, at the apical level from $9 \mathrm{~mm}$ to $4 \mathrm{~mm}$. Figure 9 shows that although the pixel intensity of the papillary muscles (the darker regions inside the green contours) and the myocardium is similar, the proposed method is able to exclude the papillary muscles from the myocardium. This is because of the attraction between the two epi- and endocardium contours enforced by the containment energy term. Figure 10 displays the result of ACWOE as well as the effect of the containment term on LV segmentation. Without the containment constraint, the level set cannot segment the left ventricle properly, Figure

\footnotetext{
${ }^{2}$ Due to different breath-holds between slice acquisition there could be misalignments between different short axis slices. Ideally, these slices must be properly aligned in a pre-processing stage prior to segmentation. However, to use the provided ground truth and for fair comparison with other methods, we use the cardiac data as provided with simple noise reduction and smoothing.
} 


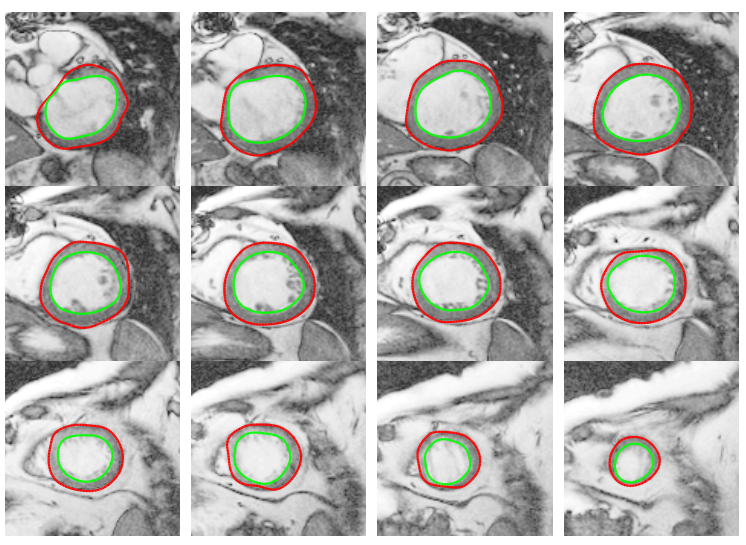

Figure 9: A representative sample of LV segmentation using our method for one subject at different slice levels: Basal (first row), mid-cavity (second row) and apical (third row).

10(b), while this issue has been addressed in Figure 10(c) via the attraction between the two red and green contours.

Since there is no applicable exclusion constraint for LV segmentation, we set $\lambda_{3}=0$ in (10). Hence, we have two free parameters to set for LV segmentation: $\lambda_{1}$ and $\lambda_{2}$ control the contribution of the regional intensity term and the containment term. From an energy minimization point of view, one of the parameters can be fixed and we have only one free parameter to set. We set $\lambda_{1}=1$ and varied $\lambda_{2}$ from 0 to 10 to find the best value in the training set provided in the Sunnybrook dataset. The obtained optimum value for $\lambda_{2}$ is 3.8 .

For initialization, we provided the initial contours (similar to Figure 10(a)) in the mid-axial cardiac slice of the 3D scan of each subject. The level sets then evolve in 3D.

We quantitatively evaluate our segmentation method based on two measures:

1) The average distance error: measures the perpendicular distance between the resultant contour and the corresponding manually drawn expert contour, averaged over all contour points. The smaller the average distance value implies that the two contours match more closely.

2) The DSC described earlier.

Tables IV and V compare the proposed method's performance with other competing methods that were evaluated on the same dataset. Figure 11 visualizes the average distance error (in $\mathrm{mm}$ ) obtained from the proposed method for all cases in the Sunnybrook validation dataset. One of the important clinical parameters for cardiac diagnosis is the left ventricular volume. The LV volume determined by the proposed method and by manual expert segmentation have been compared in Figures 12(a) and (b) for 16 different subjects (volumes) over the two ES and ED phases of the cardiac cycle. Figure 12(b), illustrates the Bland-Altman plot [36], which is used to compare two clinical measurements and shows the difference between the two measurements versus their average value. The Bland-Altman plot is useful for detecting any systematic bias between the two measurements and identifying possible outliers. The limits of agreement in a Bland-Altman analysis is usually specified as mean(difference $) \pm 1.96 \times$ std(difference),

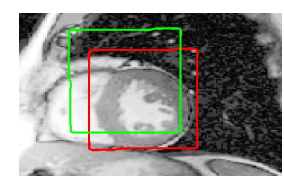

(a)

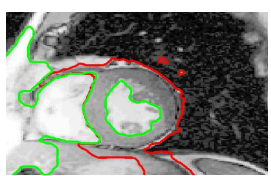

(b)

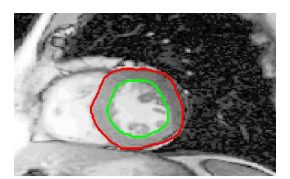

(c)
Figure 10: Effect of the containment term on LV segmentation. (a) Endocardium (green) and epicardium (red) initialization, (b) ACWOE result, (c) Our result with containment energy term. Note how the containment constraint improves the LV segmentation by creating attraction and repulsion on epi- and endocardial boundaries.

Table IV: LV segmentation results (Sunnybrook dataset): DSC

\begin{tabular}{|c|c|c|}
\hline Method & LV endo. & LV epi. \\
\hline \hline Marak et al. 2009 [35] & $0.86 \pm 0.04$ & $0.93 \pm 0.02$ \\
Casta et al. 2009 [10] & $? \pm ?$ & $0.93 \pm ?$ \\
Lu at al. 2009 [32] & $0.89 \pm 0.03$ & $0.94 \pm 0.02$ \\
Wijnhout et al. 2009 [65] & $0.89 \pm 0.03$ & $0.93 \pm 0.01$ \\
O'Brien et al. 2009 [48] & $0.81 \pm ?$ & $0.91 \pm ?$ \\
Constantinides et al. 2009 [16] & $0.89 \pm 0.04$ & $0.92 \pm 0.02$ \\
Huang et al. [25] & $0.89 \pm 0.04$ & $0.94 \pm 0.01$ \\
Jolly et al. 2009 [26] & $0.88 \pm 0.04$ & $0.93 \pm 0.02$ \\
Ulén et al. 2013 [61] & $0.86 \pm 0.05$ & $0.92 \pm 0.02$ \\
Our method & $0.90 \pm 0.03$ & $0.94 \pm 0.01$ \\
\hline
\end{tabular}

"?": Not reported in the corresponding paper

Table V: LV segmentation results (Sunnybrook dataset): Average distance error

\begin{tabular}{|c|c|c|}
\hline Method & LV endo. & LV epi. \\
\hline \hline Marak et al. 2009 [35] & $2.60 \pm 0.38$ & $3.00 \pm 0.59$ \\
Casta et al. 2009 [10] & $? \pm ?$ & $2.72 \pm ?$ \\
Lu at al. 2009 [32] & $1.91 \pm 0.63$ & $2.07 \pm 0.61$ \\
Wijnhout et al. 2009 [65] & $2.28 \pm ?$ & $2.29 \pm ?$ \\
O'Brien et al. 2009 [48] & $3.16 \pm ?$ & $3.73 \pm ?$ \\
Constantinides et al. 2009 [16] & $2.35 \pm 0.57$ & $2.04 \pm 0.47$ \\
Huang et al. [25] & $2.11 \pm 0.41$ & $2.06 \pm 0.39$ \\
Jolly et al. 2009 [26] & $1.97 \pm 0.48$ & $2.26 \pm 0.59$ \\
Ulén et al. 2013 [61] & $? \pm ?$ & $? \pm ?$ \\
Our method & $1.89 \pm 0.29$ & $1.98 \pm 0.33$ \\
\hline
\end{tabular}

"?": Not reported in the corresponding paper

where mean(.) and $s t d($.$) are the average and the standard$ deviation of the data, respectively. If the difference is within mean \pm 1.96 std then it is deemed not clinically important, i.e. the two methods (our proposed method and the expert segmentation) can be used interchangeably. The average time for segmenting one phase (ES or ED) in a single volume on a $3.4 \mathrm{GHz}$ Intel(R) CPU with $16 \mathrm{~GB}$ RAM is about 65 seconds.

2) Left and right ventricles segmentation: Studies show that the right ventricular (RV) function may be effective for diagnosing cardiovascular diseases such as pulmonary hypertension, congenital heart disease, and coronary artery disease [44]. Myocardial left and right ventricular segmentation is a suitable application for our framework since it exhibits both containment and exclusion geometrical constraints. In the cardiac model, the myocardium contains both the left and right ventricles while left and right ventricles are excluded from 


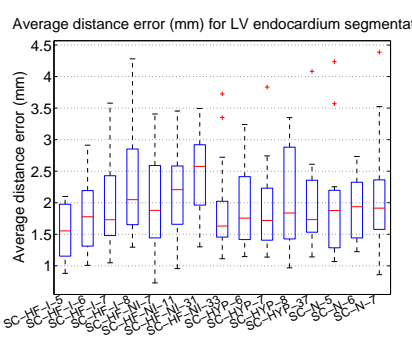

(a)

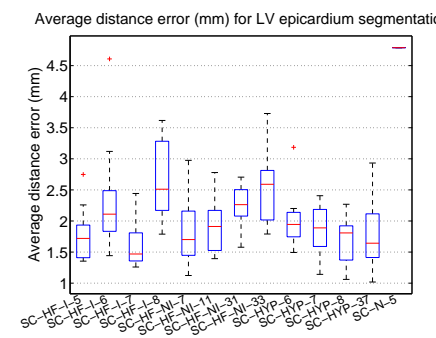

(b)
Figure 11: Box plots showing the average distance error ( $\mathrm{mm}$ ) between the obtained results and the ground truth for $\mathrm{LV}$, (a) endocardium, and (b) epicardium segmentation of the Sunnybrook dataset. Results are shows for 16 different cases (along the x-axis). The top and bottom line of each box indicate the first and third quartiles of the measurements, respectively. The red line in the middle of each box shows the median. The whiskers from each box show the largest and smallest observation and the "+" symbol show the outliers.

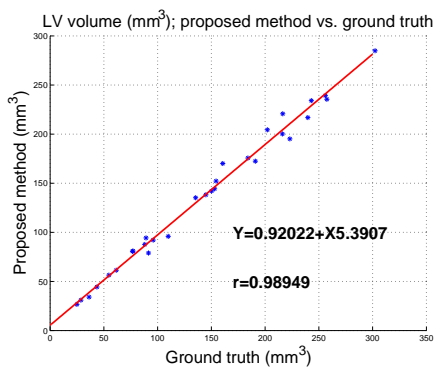

(a)

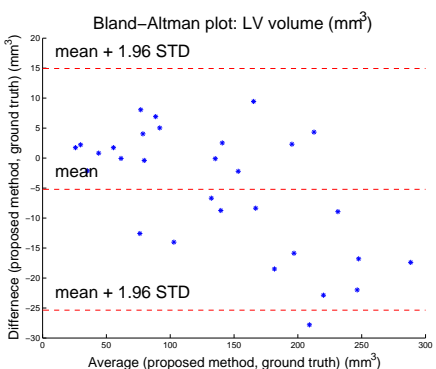

(b)
Figure 12: Scatter plots of the proposed method results against the ground truth for $\mathrm{LV}$ volume in $\mathrm{mm}^{3}$. (a) Regression analysis for LV volume measurement. (b) Bland-Altman plot comparing the proposed method and the ground truth on LV volume measurement.

one another (Figure 13(a)). We use our framework to encode these geometrical constraints and segment the whole heart as an object consisting of multiple parts.

We evaluated our method on both the Sunnybrook and the Rouen datasets. The Rouen dataset consists of 16 shortaxis cardiac MR volumes obtained using a 1.5T MRI. All the images were obtained during 10-15 second one breathhold with a temporal resolution of 20 cardiac phases over the heart cycle. The ground truth of the right ventricle endocardial and epicardial contours have been provided by Rouen University Hospital. In both these datasets, we segmented the myocardium and the left and right ventricles simultaneously. Since the Sunnybrook and the Rouen datasets provide the ground truth segmentation only for LV and RV, respectively, we only report the results for the parts for which ground truth has been provided, i.e. the LV for the Sunnybrook and RV for the Rouen dataset.

We use three simple elliptic cylinders as initialization surfaces for LV, RV and myocardium. The centres and radii of these elliptic cylinders are different for three basal, mid-cavity

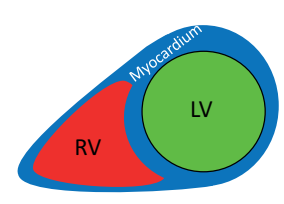

(a)

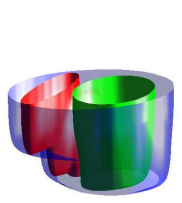

(b)

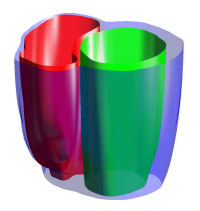

(c)

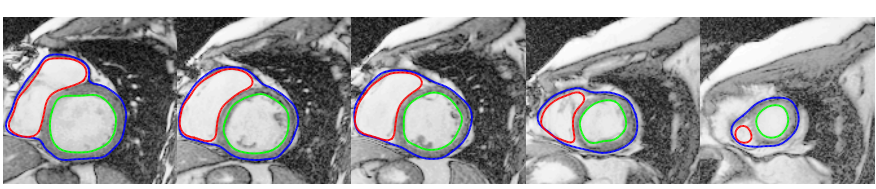

(d)

(g)

(h) (f)

Figure 13: Myocardium and left and right ventricles simultaneously segmented using the proposed geometrical constraints. (a) Cardiac model. Myocardium contains left and right ventricles, while left and right ventricles are excluded from one another. (b) 3D model used for initialization. (c) 3D rendering of the segmentation of case SC-HF-I-5 from Sunnybrook dataset. (d)-(h) 2D cross sections of the segmentation result of (c).

and apical slice levels (Figure 13(b)). In this experiment we have three distances between the endo- and epicardium in the left ventricle $\left(d_{L}\right)$, the endo- and epicardium in the right ventricle $\left(d_{R}\right)$ and, the distance between the left and right ventricles $\left(d_{L R}\right)$. Similar to LV segmentation in Section V-C1, we allow $d_{L}$ to vary from $9 \mathrm{~mm}$ to $4 \mathrm{~mm}$ from the basal to the apical level. In this experiment we set $d_{R}=4 \mathrm{~mm}$ and $d_{L R}=6 \mathrm{~mm}$. To have a suitable estimation of the weights $\lambda_{1}, \lambda_{2}$ and $\lambda_{3}$ in (10), we tune these weights using the leaveone-out cross validation technique over the dataset.

Figure 13(d)-(h) illustrates qualitative cardiac segmentation results for the case SC-HF-I-5 in the Sunnybrook dataset and Figure 13(c) shows its corresponding 3D rendering. Due to the regularization term in our level-set formulation, the RV insertion points might be over smoothed. This issue can be addressed by post-processing or by imposing a spatiallyvarying regularization into the level set framework. Figures 14(a) and (b) show the linear regression analysis for LV and $\mathrm{RV}$ area $\left(\mathrm{mm}^{2}\right)$ respectively, obtained by the proposed method and the ground truth for each single slice of ED and ES phases in the Sunnybrook and Rouen volumes. The correlation value between the proposed method and the ground truth is 0.963 and 0.978 for LV and RV segmentation, respectively. Also, the Bland-Altman plot for LV and RV segmentation are shown in Figures 14(c) and (d).

Note that while in our segmentation approach we segment the full myocardium (Figure 13), we only compare the LV and $\mathrm{RV}$ endocardium with the ground truth and not the epicardium, since the two Sunnybrook and Rouen datasets have provided ground truth for only the LV and RV epicardium, respectively. Table VI reports the DSC and average distance error for simultaneous LV and RV segmentation. 
Table VI: Simultaneous LV and RV endocardium segmentation results

\begin{tabular}{c|c|c}
\hline Region & DSC & Average distance error $(\mathrm{mm})$ \\
\hline Left ventricle & $0.89 \pm 0.03$ & $2.15 \pm 0.41$ \\
Right ventricle & $0.87 \pm 0.02$ & $1.79 \pm 0.41$ \\
\hline
\end{tabular}

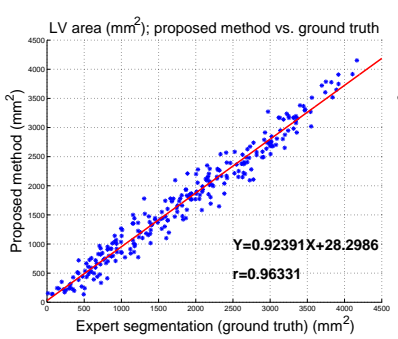

(a)

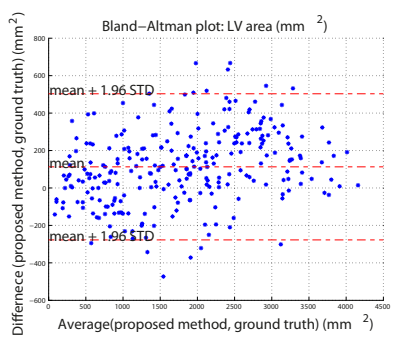

(c)

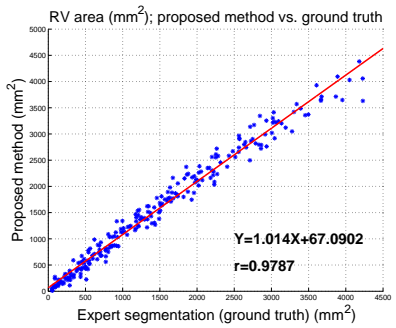

(b)

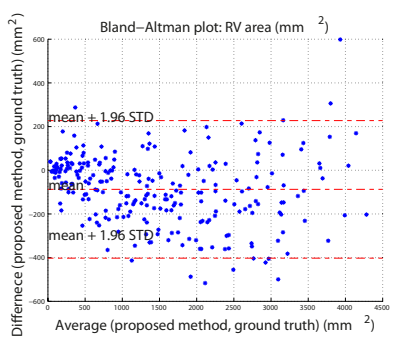

(d)
Figure 14: Scatter plots of the proposed method against the manual segmentation (ground truth) for LV and RV area measurement in $\mathrm{mm}^{2}$. Regression and correlation analysis of the area of (a) LV from the Sunnybrook dataset, and (b) RV from Rouen training dataset in two ED and ES cardiac phases. Bland-Altman plot for (c) LV area and (d) RV area measurement.

\section{Brain dynamic-PET segmentation}

To test our framework on more complex application, we applied our method to dynamic positron emission tomography (dPET) images, where, at each pixel in the image, a time activity curve describes the metabolic activity of a tissue as a result of tracer uptake, Figure 15(a). Figure 15 shows an example of segmenting a dPET image, $I: \Omega \subset \mathbb{R}^{2} \rightarrow \mathbb{R}^{40}$. Note the low signal-to-noise ratio (SNR) of the dPET image (Figure 15(b)), which is the result of not having enough time to collect a large number of photons within the short time intervals needed to capture the tracer kinematics. Our spatial relationships include: 1) the skull contains gray matter; 2) gray matter contains white matter; 3) white matter contains putamen; 4) putamen and cerebellum must be excluded from one another. From Figure 15, the problems of putamen surrounding cerebellum (yellow around green), mentioned in [54], are now clearly solved (Figure 15(h)). Despite the low signal to noise ratio in the dPET image and with a not great initialization (Figure 15(d)), our method's ability to incorporate geometric constraints results in an improved and anatomically plausible segmentation compared to the results reported in (e) and (f). We also compared our method with and without the exclusion

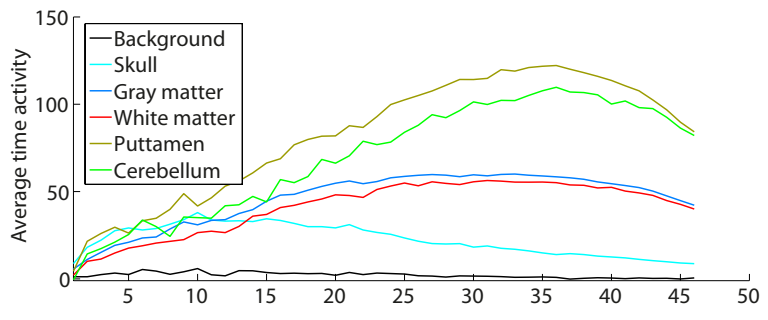

(a)

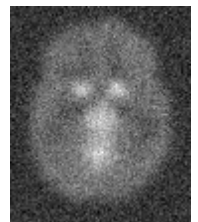

(b)

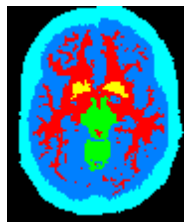

(c)

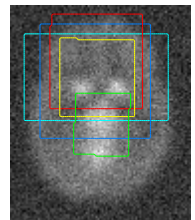

(d)

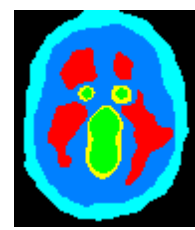

(e)

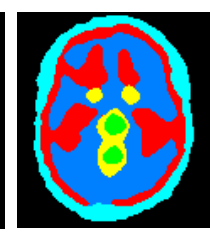

(f)

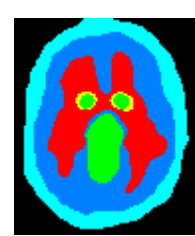

(g)

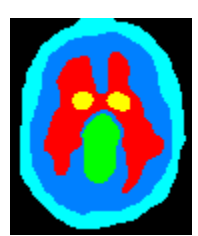

(h)
Figure 15: Brain dPET segmentation. (a) Average TAC for each functional region in the ground truth. (b) Raw image (last frame of the dynamic sequence, which is typically visualized by clinicians). (c) Ground truth. (d) Initialization. (e) Multiphase ACWOE (no constraints). (f) Saad et al. [54]. (g) Our result with containment constraint but without enforcing any exclusion constraint. (h) Our result with containment and exclusion constraint. Note how the putamen is contained by the white matter (red) as it should be whereas (e) and (f) are anatomically incorrect. Also, note how the putamen (yellow) and cerebellum (green) are properly detached in (h) as opposed to $(\mathrm{e}-\mathrm{g})$.

constraint. As shown in Figure 15(g), without the exclusion constraint, we still can get the incorrect result of putamen surrounding cerebellum (yellow around green) due to their TAC similarity. By enforcing the exclusion constraint between putamen and cerebellum, we ensure that the final result is anatomically plausible (Figure 15(h)). We emphasize that a bad and irrational initialization will result in a wrong segmentation due to our local optimization framework. We empirically set $\lambda_{1}, \lambda_{2}$ and $\lambda_{3}$ to $0.68,0.04$ and 0.15 , respectively, to balance between data, containment and exclusion terms. In fact, here, a very small weight for geometric constraint was enough to place the contours in the correct ordering to satisfy the geometric constraints.

\section{A NOTE ON CONTAINMENT CONSTRAINT Without ATTRACTION FORCES}

In this section, we discuss a special case of containment constraint in our framework with a corresponding result on real data. In the proposed framework, by having equation (1) (and its extended version (7)), there is always interactions 


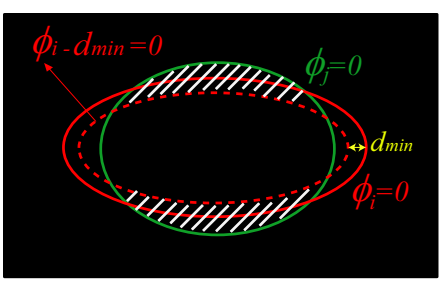

(a)

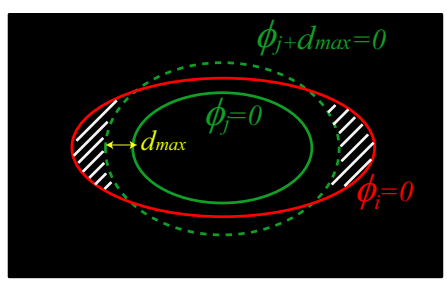

(b)

Figure 16: Enforcing (a) minimum and (b) maximum distance between regions $i$ and $j$ using (17) while $i$ contains $j$. The shaded area shows the region that is penalized by (17).

(attraction/repulsion) between the surfaces with containment constraint. There are cases in which the attraction between two regions with containment constraint is not important, e.g. there might be several small disjoint regions $j$ contained by region $i$. To address this case, we replace (1) with the following modified energy term for the case in which $i$ contains $j$ :

$$
E_{C}^{N D}\left(\phi_{i}, \phi_{j}\right)=\int_{\Omega} H\left(-\phi_{i}(x)\right) H\left(\phi_{j}(x)\right) d x .
$$

The way the above equation works is similar to the exclusion equation (3). $E_{C}^{N D}$ penalizes region $j$ that falls outside region $i$. Thanks to the level set's nature, we can enforce minimum and maximum distance (but without attraction/repulsion forces) between regions' boundaries by modifying (16) as follows

$$
\begin{aligned}
E_{C}^{N D}\left(\phi_{i}, \phi_{j}\right) & =\int_{\Omega} H\left(-\phi_{i}(x)+d_{\min }\right) H\left(\phi_{j}(x)\right) d x \\
& +\int_{\Omega} H\left(\phi_{i}(x)\right) H\left(-\phi_{j}(x)-d_{\max }\right) d x,
\end{aligned}
$$

where the first term enforces minimum distance of $d_{\text {min }}$ pixels between two $i$ and $j$ regions and the second term ensures that $i$ does not grow farther than $d_{\max }$ pixels from $j$ 's boundary. The term $H\left(\phi_{i}-d_{m i n}\right)$ corresponds to the shrunk version of $i$ by $d_{\min }$ pixels. The first term in (17) penalizes region $j$ that falls outside the shrunk $i$. On the other hand, the term $H\left(\phi_{j}+d_{\max }\right)$ expand the zero level set of $\phi_{j}$ by $d_{\max }$ pixels. The second term in (17) penalizes region $i$ that falls outside the expanded $j$. Figure 16 shows the mechanism of how (17) works.

One practical example is lung blood vessels segmentation. In this case, blood vessels have to be contained in the lungs probably without any specific distance constraint between them. Here, we set $d_{\min }=1$ pixel. In this example, the lung stands out with high contrast and it is unlikely that the corresponding surface grow irrationally far from the blood vessels. Hence, we ignored the maximum distance (one may set $d_{\max }$ arbitrary large). Figure 17 shows 3D blood vessel segmentation in a lung. Figure 17(a) shows an unbiased initialization. Note how the incorrect segmentation in Figures 17(b) and (c) performed by ACWOE (without containment constraint) has been improved by our framework using (17) as the containment energy term (Figures 17(d) and (e)).

\section{CONCLUSIONS}

In this work, we augmented the level set framework with two important geometric constraints, containment and exclu-

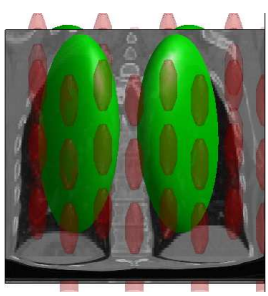

(a)

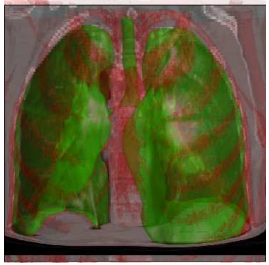

(b)

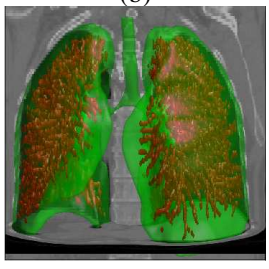

(d)

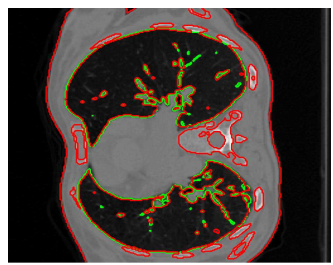

(c)

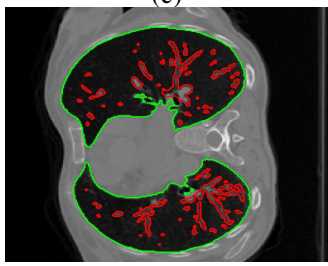

(e)
Figure 17: Lung and its blood vessels segmentation. (a) Initialization. (b) 3D ACWOE result. (c) ACWOE segmentation result in a $2 \mathrm{D}$ slice. (d) The proposed method result using (17) as the containment term. (e) The proposed method's result shown in a $2 \mathrm{D}$ slice.

sion, along with a distance prior for segmenting spatiallyrecurring multi-region objects. We showed that only adding the containment and exclusion terms into the level set framework can improve the segmentation results in a number of applications, even when only a simple intensity/color-based data term is used.

By comparing our local optimization-based framework in the continuous domain with its counterpart methods in the discrete domain [18], [61], we draw the following conclusions: 1) Metrication error: This issue might not be an overwhelming issue in many medical applications, however, it remains a known issue with discrete (graph-based) methods, which makes it difficult to efficiently implement a convex regularizer like total variation (TV) in the discrete domain. On the other hand, due to our continuous formulation, our method is free from metrication error. 2) Memory usage: Due to the graphical representation of an image, the graph based methods, e.g. [18] and [61], consume more memory compared with our framework. This also is due to the fact that [18] and [61] explore the whole search space to find the global solution as opposed to our method that only finds a locally optimal solution. 3) Runtime: In general, global graph based methods are highly efficient in finding the optimal solution. However, the computation time and memory usage in these methods, [18] and [61], depend intricately on the distance (thickness) constraint between regions due to the need for adding extra edges per pixel. The runtime and memory usage in our framework, on the other hand, is almost constant with 
respect to different distance constraints rendering our method suitable for segmenting very large microscopy images (100s of mega-pixels). 4) Initialization and numerical stability: While our method's results depend on the initialization and needs to satisfy the CFL condition for numerical stability, graph based methods avoid such requirements and have proven to be numerically stable. Nevertheless, we showed favourable results even with fully automatic or rough initialization that are distant from the desired boundaries.

As future work, we see two directions: adding more priors, e.g. shape, to further improve the descriptiveness of the objective function of the current framework as well as exploring ways to improve optimizability (e.g. proposing convex forms for such constraints) while minimizing the effect on the data fidelity term.

\section{ACKNOWLEDGMENT}

The authors would like to thank the reviewers and editors of TMI for their constructive feedback. This work has been partially supported by the Natural Sciences and Engineering Research Council of Canada (NSERC).

\section{REFERENCES}

[1] Y. Al-Kofahi, W. Lassoued, W. Lee, and B. Roysam. Improved automatic detection and segmentation of cell nuclei in histopathology images. IEEE Trans. Biomed. Eng., 57(4):841-852, 2010.

[2] K. Alahari, C. Russell, and P.H.S. Torr. Efficient piecewise learning for conditional random fields. In IEEE CVPR, pages 895-901, 2010.

[3] S. Andrews, G. Hamarneh, A. Yazdanpanah, B. HajGhanbari, and W. Darlene Reid. Probabilistic multi-shape segmentation of knee extensor and flexor muscles. In MICCAI, volume 6893, pages 651-658, 2011.

[4] S. Andrews, C. McIntosh, and G. Hamarneh. Convex multi-region probabilistic segmentation with shape prior in the isometric log-ratio transformation space. In IEEE ICCV, pages 2096-2103, 2011.

[5] Elsa D Angelini, Ting Song, Brett D Mensh, and Andrew Laine. Multiphase three-dimensional level set segmentation of brain mri. In MICCAI, pages 318-326. 2004.

[6] Ismail Ben Ayed, Shuo Li, Ali Islam, Greg Garvin, and Rethy Chhem. Area prior constrained level set evolution for medical image segmentation. In SPIE, volume 6914, page 691402, 2008.

[7] Ismail Ben Ayed, Shuo Li, and Ian Ross. A statistical overlap prior for variational image segmentation. Int. J. Comput. Vis., 85(1):115-132, 2009.

[8] Yuri Boykov and Vladimir Kolmogorov. Computing geodesics and minimal surfaces via graph cuts. In IEEE ICCV, pages 26-33, 2003.

[9] Xavier Bresson, Pierre Vandergheynst, and Jean-Philippe Thiran. A variational model for object segmentation using boundary information and shape prior driven by the mumford-shah functional. Int. J. Comput. Vis., 68:145-162, 2006.

[10] C. Casta, P. Clarysse, J. Schaerer, and J. Pousin. Evaluation of the dynamic deformable elastic template model for the segmentation of the heart in mri sequences. The MIDAS Journal, Cardiac MR Left Ventricle Segmentation Challenge, London, UK, 2009.

[11] Sunnybrook Health Sciences Centre. Cardiac MR left ventricle segmentation challenge. MICCAI, 2009.

[12] T.F. Chan, B.Y. Sandberg, and L.A. Vese. Active contours without edges for vector-valued images. J. Visual Commun. Image Represent., 11(2):130-141, 2000.

[13] T.F. Chan and L.A. Vese. Active contours without edges. IEEE Trans. Image Process., 10(2):266-277, 2001.

[14] D.L. Collins and A.C. Evans. Animal: validation and applications of nonlinear registration-based segmentation. Int. J. Pattern Recognit Artif Intell., 11(8):1271-1294, 1997.

[15] Colliot, Camara, and Bloch. Integration of fuzzy spatial relations in deformable models-application to brain MRI segmentation. Pattern Recognit., 39(8):1401-1414, 2006.
[16] C. Constantinides, Y. Chenoune, N. Kachenoura, E. Roullot, E. Mousseaux, A. Herment, and F. Frouin. Semi-automated cardiac segmentation on cine magnetic resonance images using gvf-snake deformable models. The MIDAS Journal-Cardiac MR Left Ventricle Segmentation Challenge, 2009.

[17] Richard Courant, Kurt Friedrichs, and Hans Lewy. On the partial difference equations of mathematical physics. IBM journal of Research and Development, 11(2):215-234, 1967.

[18] A. Delong and Y. Boykov. Globally optimal segmentation of multiregion objects. In IEEE ICCV, pages 285-292, 2009.

[19] Felzenszwalb and Veksler. Tiered scene labeling with dynamic programming. In IEEE CVPR, pages 3097-3104, 2010.

[20] J.C. Gee, M. Reivich, and R. Bajcsy. Elastically deforming a threedimensional atlas to match anatomical brain images1. J. Comput. Assisted Tomogr., 17:225-236, 1993.

[21] Roman Goldenberg, Ron Kimmel, Ehud Rivlin, and Michael Rudzsky. Cortex segmentation: A fast variational geometric approach. IEEE Trans. Med. Imaging, 21(12):1544-1551, 2002.

[22] S. Gould, J. Rodgers, D. Cohen, G. Elidan, and D. Koller. Multi-class segmentation with relative location prior. IJCV, 80(3):300-316, 2008.

[23] G. Hamarneh. Multi-label mrf optimization via a least squares s-t cut. In ISVC OVGMI, volume 5875-I, pages 1055-1066, 2009.

[24] Rouen University Hospital. RV segmentation challenge in cardiac MRI, http://www.litislab.eu/rvsc. MICCAI, 2012.

[25] S. Huang, J. Liu, L. Lee, S. Venkatesh, L. Teo, C. Au, and W. Nowinski. Segmentation of the left ventricle from cine MR images using a comprehensive approach. In International Conference on Functional Imaging and Modeling of the Heart, pages 339-347, 2009.

[26] M. Jolly. Fully automatic left ventricle segmentation in cardiac cine mr images using registration and minimum surfaces. The MIDAS JournalCardiac MR Left Ventricle Segmentation Challenge, 2009.

[27] V. Kolmogorov, Y. Boykov, and C. Rother. Applications of parametric maxflow in computer vision. In IEEE ICCV, pages 1-8, 2007.

[28] K. Li, X. Wu, D.Z. Chen, and M. Sonka. Optimal surface segmentation in volumetric images-a graph-theoretic approach. IEEE Trans. Pattern Anal. Mach. Intell., 28(1):119-134, 2006.

[29] Shuo Li, Thomas Fevens, Adam Krzyżak, Chao Jin, and Song Li. Fast and robust clinical triple-region image segmentation using one level set function. In MICCAI, pages 766-773. 2006.

[30] A. Litvin and W. Karl. Coupled shape distribution-based segmentation of multiple objects. In IEEE Trans. Pattern Anal. Mach. Intell., pages 171-233, 2005

[31] X. Liu, O. Veksler, and J. Samarabandu. Graph cut with ordering constraints on labels and its applications. In IEEE CVPR, pages 1-8, 2008.

[32] Y. Lu, P. Radau, K. Connelly, A. Dick, and G. Wright. Automatic imagedriven segmentation of left ventricle in cardiac cine MRI. The MIDAS Journal, 49, 2009.

[33] A.R. Mansouri, A. Mitiche, and C. Vázquez. Multiregion competition: A level set extension of region competition to multiple region image partitioning. Comput. Vision Image Understanding, 101(3):137-150, 2006.

[34] KZ Mao, P. Zhao, and P.H. Tan. Supervised learning-based cell image segmentation for p53 immunohistochemistry. IEEE Trans. Biomed. Eng., 53(6):1153-1163, 2006.

[35] L. Marak, J. Cousty, L. Najman, H. Talbot, et al. 4D Morphological segmentation and the MICCAI LV-segmentation grand challenge. In MICCAI Workshop on Cardiac MR Left Ventricle Segmentation Challenge. MIDAS Journal, 2009.

[36] J. Martin Bland and D.G. Altman. Statistical methods for assessing agreement between two methods of clinical measurement. The lancet, 327(8476):307-310, 1986.

[37] T. McInerney, G. Hamarneh, M. Shenton, and D. Terzopoulos. Deformable organisms for automatic medical image analysis. Med. Image Anal., 6(3):251-266, 2002.

[38] C. McIntosh and G. Hamarneh. Is a single energy functional sufficient? adaptive energy functionals and automatic initialization. MICCAI, pages 503-510, 2007.

[39] C. McIntosh and G. Hamarneh. Is a single energy functional sufficient? adaptive energy functionals and automatic initialization. In MICCAI, volume 4792, pages 503-510, 2007.

[40] C. McIntosh and G. Hamarneh. Medial-based deformable models in nonconvex shape-spaces for medical image segmentation. IEEE Trans. Med. Imaging, 31(1):33-50, 2012.

[41] Amar Mitiche and Ismail Ben Ayed. Variational and level set methods in image segmentation, volume 5. Springer, 2010. 
[42] C. Nambakhsh, J. Yuan, K. Punithakumar, A. Goela, M. Rajchl, T.M Peters, and I. Ben Ayed. Left ventricle segmentation in MRI via convex relaxed distribution matching. Med. image anal., 17(8):1010-1024, 2013.

[43] Claudia Nieuwenhuis, Eno Töppe, and Daniel Cremers. A survey and comparison of discrete and continuous multi-label optimization approaches for the potts model. Int. J. Comput. Vis., 104(3):223-240, 2013.

[44] P.A. Noseworthy, C. Newton-Cheh, F.J. Schoen, M. Naik, D. Kim, F. O'Brien, L. Axel, and M.B. Srichai. Contemporary reviews in cardiovascular medicine. Circulation, 118(18), 2008.

[45] M.S. Nosrati, S. Andrews, and G. Hamarneh. Bounded labeling function for global segmentation of multi-part objects with geometric constraints. In IEEE ICCV, pages 2032-2039, 2013.

[46] M.S. Nosrati and G. Hamarneh. Segmentation of cells with partial occlusion and part configuration constraint using evolutionary computation. In MICCAI, volume 8149, pages 461-468, 2013.

[47] S. Nowozin, P. Gehler, and C. Lampert. On parameter learning in crfbased approaches to object class image segmentation. ECCV, pages 98-111, 2010

[48] S. OBrien, O. Ghita, and P. Whelan. Segmenting the left ventricle in $3 d$ using a coupled asm and a learned non-rigid spatial model. The MIDAS Journal, 49, 2009.

[49] Nikos Paragios. A variational approach for the segmentation of the left ventricle in cardiac image analysis. Int. J. Comput. Vis., 50(3):345-362, 2002.

[50] C. Pluempitiwiriyawej, J.M.F. Moura, Y.J.L. Wu, and C. Ho. Stacs: New active contour scheme for cardiac MR image segmentation. IEEE Trans. Med. Imaging, 24(5):593-603, 2005.

[51] Martin Rajchl, Jing Yuan, James A White, Cyrus MS Nambakhsh, Eranga Ukwatta, Feng Li, John Stirrat, and Terry M Peters. A fast convex optimization approach to segmenting 3D scar tissue from delayedenhancement cardiac MR images. In MICCAI, pages 659-666. 2012.

[52] J. Rao, R. Abugharbieh, and G. Hamarneh. Adaptive regularization for image segmentation using local image curvature cues. In $E C C V$, pages 651-665, 2010

[53] C. Rother, P. Kohli, W. Feng, and J. Jia. Minimizing sparse higher order energy functions of discrete variables. In IEEE CVPR, pages 1382-1389, 2009.

[54] A. Saad, G. Hamarneh, T. Möller, and B. Smith. Kinetic modeling based probabilistic segmentation for molecular images. MICCAI, pages 244-252, 2008.

[55] Christophe Samson, Laure Blanc-Féraud, Gilles Aubert, and Josiane Zerubia. A level set model for image classification. Int. J. Comput. Vis., 40(3):187-197, 2000.

[56] Frank R Schmidt and Yuri Boykov. Hausdorff distance constraint for multi-surface segmentation. In ECCV, pages 598-611. 2012.

[57] T. Shen, H. Li, and X. Huang. Active volume models for medical image segmentation. IEEE Trans. Med. Imaging, 30(3):774-791, 2011.

[58] E. Strekalovskiy and D. Cremers. Generalized ordering constraints for multilabel optimization. In IEEE ICCV, pages 2619-2626, 2011.

[59] M. Szummer, P. Kohli, and D. Hoiem. Learning crfs using graph cuts. ECCV, pages 582-595, 2008.

[60] Eranga Ukwatta, Jing Yuan, Martin Rajchl, and Aaron Fenster. Efficient global optimization based 3D carotid AB-LIB MRI segmentation by simultaneously evolving coupled surfaces. In MICCAI, pages 377-384. 2012.

[61] J. Ulen, P. Strandmark, and F. Kahl. An efficient optimization framework for multi-region segmentation based on lagrangian duality. IEEE Trans. Med. Imaging, PP(99):1, 2013.

[62] A. Vazquez-Reina, E. Miller, and H. Pfister. Multiphase geometric couplings for the segmentation of neural processes. In IEEE CVPR, pages 2020-2027, 2009.

[63] L.A. Vese and T.F. Chan. A multiphase level set framework for image segmentation using the Mumford and Shah model. Int. J. Comput. Vis., 50(3):271-293, 2002.

[64] Z. Wang and B. Vemuri. Tensor field segmentation using region based active contour model. ECCV, pages 304-315, 2004.

[65] J.S. Wijnhout, D. Hendriksen, H.C. Assen, and R.J. Geest. LV challenge lkeb contribution: Fully automated myocardial contour detection. Midas Journal, page 683, 2009.

[66] X. Wu, M. Amrikachi, and S.K. Shah. Embedding topic discovery in conditional random fields model for segmenting nuclei using multispectral data. IEEE Trans. Biomed. Eng., 59(6):1539-1549, 2012.

[67] X. Wu, X. Dou, A. Wahle, and M. Sonka. Region detection by minimizing intraclass variance with geometric constraints, global optimality, and efficient approximation. IEEE Trans. Med. Imaging, 30(3):814-827, 2011.

[68] L. Yang, O. Tuzel, P. Meer, and D. Foran. Automatic image analysis of histopathology specimens using concave vertex graph. MICCAI, pages 833-841, 2008.

[69] A. Yazdanpanah, G. Hamarneh, B. Smith, and M. Sarunic. Automated segmentation of intra-retinal layers from optical coherence tomography images using an active contour approach. IEEE Trans. Med. Imaging, 30(2):484-496, 2011.

[70] Xiaolan Zeng, Lawrence H Staib, Robert T Schultz, and James S Duncan. Volumetric layer segmentation using coupled surfaces propagation. In IEEE CVPR, pages 708-715, 1998.

[71] H.K. Zhao, T. Chan, B. Merriman, and S. Osher. A variational level set approach to multiphase motion. J. Comput. Phys., 127(1):179-195, 1996. 